\title{
S-sulfocysteine/NMDA receptor-dependent signaling underlies neurodegeneration in molybdenum cofactor deficiency
}

\author{
Avadh Kumar, ${ }^{1}$ Borislav Dejanovic, ${ }^{1}$ Florian Hetsch, ${ }^{2}$ Marcus Semtner, ${ }^{3}$ Debora Fusca, ${ }^{4}$ Sita Arjune, ${ }^{1}$ \\ Jose Angel Santamaria-Araujo, ${ }^{1}$ Aline Winkelmann, ${ }^{2,4}$ Scott Ayton, ${ }^{5}$ Ashley I. Bush, ${ }^{5}$ Peter Kloppenburg, ${ }^{4,6}$ Jochen C. Meier, ${ }^{2}$ \\ Guenter Schwarz, ${ }^{1,6}$ and Abdel Ali Belaidi ${ }^{1,5}$ \\ IInstitute of Biochemistry, Department of Chemistry, Center for Molecular Medicine Cologne (CMMC), University of Cologne, Cologne, Cermany. ${ }^{2}$ TU Braunschweig, Zoological Institute, Division of Cell \\ Physiology, Braunschweig, Cermany. ${ }^{3}$ Cellular Neurosciences, Max Delbrück Center for Molecular Medicine in the Helmholtz Association, Berlin, Cermany. ${ }^{4}$ Biocenter, Institute for Zoology, University of \\ Cologne, Cologne, Germany. ${ }^{5}$ The Florey Institute for Neuroscience and Mental Health, The University of Melbourne, Parkville, Victoria, Australia. ${ }^{6}$ Cologne Excellence Cluster on Cellular Stress Responses in \\ Ageing-Associated Diseases (CECAD), University of Cologne, Cologne, Germany.
}

\begin{abstract}
Molybdenum cofactor deficiency (MoCD) is an autosomal recessive inborn error of metabolism characterized by neurodegeneration and death in early childhood. The rapid and progressive neurodegeneration in MoCD presents a major clinical challenge and may relate to the poor understanding of the molecular mechanisms involved. Recently, we reported that treating patients with cyclic pyranopterin monophosphate (cPMP) is a successful therapy for a subset of infants with MoCD and prevents irreversible brain damage. Here, we studied S-sulfocysteine (SSC), a structural analog of glutamate that accumulates in the plasma and urine of patients with MoCD, and demonstrated that it acts as an $\mathrm{N}$-methyl D-aspartate receptor (NMDA-R) agonist, leading to calcium influx and downstream cell signaling events and neurotoxicity. SSC treatment activated the protease calpain, and calpain-dependent degradation of the inhibitory synaptic protein gephyrin subsequently exacerbated SSC-mediated excitotoxicity and promoted loss of GABAergic synapses. Pharmacological blockade of NMDA-R, calcium influx, or calpain activity abolished SSC and glutamate neurotoxicity in primary murine neurons. Finally, the NMDA-R antagonist memantine was protective against the manifestation of symptoms in a tungstate-induced MoCD mouse model. These findings demonstrate that SSC drives excitotoxic neurodegeneration in MoCD and introduce NMDA-R antagonists as potential therapeutics for this fatal disease.
\end{abstract}

\section{Introduction}

Molybdenum cofactor deficiency (MoCD) is a rare metabolic disorder $(1,2)$ characterized by severe neurological abnormalities including intractable neonatal seizures, feeding difficulties, developmental delay, ocular lens dislocation, and death in early childhood (3). More than 100 cases have been reported $(2,4,5)$, the vast majority of which have been characterized by a severe neurological phenotype (6). However, because of a lack of diagnosis or misdiagnosis $(7,8)$, the global incidence of MoCD is probably underestimated, as the initial neurological symptoms of MoCD resemble a broad spectrum of metabolic and hypoxic encephalopathies $(6,9)$.

Patients with MoCD harbor mutations in the genes encoding the enzymes molybdenum cofactor synthesis 1 (MOCS1), $M O C S 2$, or gephyrin (GPHN) that are required for molybdenum cofactor (Moco) biogenesis $(4,10)$, a pathway that is highly conserved throughout evolution. MoCD leads to the loss of activity of 4 molybdenum enzymes in humans (11) including sulfite oxidase (SO). SO is a mitochondrial enzyme (12) that catalyzes the

Conflict of interest: The authors have declared that no conflict of interest exists Submitted: August 5, 2016; Accepted: September 26, 2017.

Reference information: / Clin Invest. 2017;127(12):4365-4378.

https://doi.org/10.1172/JCl89885. oxidation of toxic sulfite, generated from cysteine metabolism, to sulfate (10). The latter is required for multiple biochemical reactions including the sulfation of several substrates important for brain development $(13,14)$. MoCD has a clinical presentation similar to that of SO deficiency (SOD) disorder (15), implicating sulfite toxicity as the major pathomechanism in both diseases (10). Patients with MoCD have high levels of sulfite in their urine, which is accompanied by elevated urinary levels of taurine and thiosulfate, while their plasma cystine level is depleted $(10,16)$. In addition, S-sulfocysteine (SSC), a secondary sulfurcontaining metabolite, accumulates in the urine and plasma of MoCD patients and is used for MoCD diagnostics $(10,17)$.

Until recently, MoCD has been a fatal disease with a median life expectancy of 3 years (18). We introduced cyclic pyranopterin monophosphate (cPMP), the biosynthetic intermediate of Moco, as a new therapy for patients with MOCS1 mutations (19). Treatment with CPMP normalized biomarkers and arrested neurodegeneration in a mouse model and in MoCD patients (19-21). In our recent prospective study (19), a remarkable clinical benefit was observed in patients treated soon after birth, who exhibited near-normal development. Treatment was less effective in patients who received delayed treatment (days to weeks after the manifestation of neurodegenerative symptoms) (19). While cPMP was effective in treating MoCD patients with MOCS1 
mutations, alternative treatments are needed for patients with mutations in MOCS2 and GPHN (22), who have normal cPMP synthesis but impairment of subsequent steps of Moco synthesis.

In this study, we investigated the influence of MoCD metabolites on neurodegeneration and identified SSC as the main factor causing neuronal cell death. We provide in vitro and in vivo evidence that suppression of either SSC-mediated NMDA-R activation or subsequent downstream signaling events rescued SSC-triggered neurotoxicity. Furthermore, we demonstrate that SSC/NMDA-R/calcium signaling resulted in calpain-dependent cleavage of the postsynaptic scaffolding protein gephyrin, causing a destabilization of GABAergic synapses - which are the target of the anticonvulsive therapies currently used in MoCD (10, 21). Therefore, our study provides a molecular understanding of the underlying pathomechanism that can be responsible for the intractable nature of seizures in patients with MoCD and provides a therapeutic strategy for symptomatic seizure control.

\section{Results}

Sulfite and SSC induce toxicity in cortical neurons. Sulfite, thiosulfate, taurine, and SSC levels are increased in the urine of patients with MoCD because of SO inactivity, while cysteine levels are reduced (10). We first explored whether the accumulated sulfurcontaining metabolites (S-metabolites) induce neurotoxicity in primary murine cortical neurons. We compared the toxicities of the S-metabolites to that of glutamate, since SSC was suggested to cause neurotoxicity in MoCD (23), given its structural similarity to glutamate (Figure 1A). After a 12-hour exposure, we used 2 different assays to detect cell viability (MTT assay) and cell death (propidium iodide staining), which produced consistent results. Sulfite and SSC showed dose-dependent toxicity in cortical neurons with $\mathrm{LD}_{50}$ values of $100 \pm 3 \mu \mathrm{M}$ and $74 \pm 4 \mu \mathrm{M}$, respectively, which was similar to that of glutamate $(82 \pm 2 \mu \mathrm{M})$ (Figure 1, B and $\mathrm{C})$. Taurine was not toxic, in agreement with its proposed protective role in the CNS $(24,25)$, while thiosulfate-mediated toxicity was low and only detected at high concentrations $\left(\mathrm{LD}_{50}=621 \pm 12\right.$ $\mu \mathrm{M})$. The toxicity of sulfite and SSC in cortical neurons was additionally confirmed using live/dead staining (Figure 1D and Supplemental Figure 1; supplemental material available online with this article; https://doi.org/10.1172/JCI89885DS1). In contrast to neurons, in HEK293 cells, only sulfite was effective in inducing cell death (Figure 1D and Supplemental Figure 1), suggesting that SSC is not toxic per se but, like glutamate, acts specifically on glutamatergic receptors.

SSC toxicity in MoCD involves NMDA-R activation. Early studies in rats showed that SSC, when administered intracerebrally, induces brain damage similar to that induced by glutamate (23). Given its structural similarity to glutamate, SSC was suggested to be neurotoxic (23), but this has yet to be explored as the underlying cause of neurotoxicity in MoCD. We studied whether glutamatergic signaling by SSC mediates neurotoxicity in cortical neurons and compared this with other S-metabolites, in the absence and presence of the selective NMDA-R and $\alpha$-amino-3-hydroxy-5methyl-4-isoxazolepropionic acid receptor (AMPA-R) antagonists MK801 and NBQX, respectively. SSC-induced toxicity, like glutamate toxicity, was abolished by the NMDA-R blocker MK801, but not by the AMPA-R blocker NBQX (Figure 1, E and F). Sur- prisingly, sulfite toxicity was also partially reversed by NMDA-R blockers (Figure 1, E and F). We hypothesized that sulfite reacted with cysteine and was converted to SSC in the culture medium. Under oxygenated conditions such as in serum and plasma, cysteine is converted to its oxidized cystine form, while cellular reducing conditions favor the presence of the reduced cysteine form. We probed cystine and cysteine reaction with sulfite and found a cystine- and sulfite-dependent formation of SSC in a stoichiometric manner, while cysteine was not able to form SSC with sulfite (Supplemental Figure 2A), which supports the proposed extracellular scavenging function of cysteine (10). Likewise, the addition of sulfite to neuronal culture medium resulted in a dosedependent formation of SSC, which reached saturation as a result of a limited cystine concentration in the medium (Supplemental Figure 2B). When the toxicity of sulfite and SSC to cortical neurons was assessed in cystine-containing (normal neuronal culture media) and cystine-free artificial cerebrospinal fluid (ACSF), we found that sulfite toxicity was reduced ( $58 \%$ cell viability) compared with that of glutamate and SSC (34\% and 33\% cell viability, Figure $1 \mathrm{G}$ ), and, in contrast to glutamate and SSC toxicity, MK801 completely failed to rescue sulfite-mediated toxicity (Figure 1, E and F). Thus, sulfite itself is cytotoxic, as reported earlier (26), but develops more aggressive NMDA-R-dependent neurotoxicity upon reaction with cystine by forming SSC.

SSC evokes large tonic currents and calcium influx in cultured neurons. Given the structural and toxicological similarities of SSC and glutamate, we next investigated whether SSC could functionally affect glutamatergic signaling. Voltage-clamp recordings of cultured primary hippocampal neurons (Figure 2, A and B) demonstrated that SSC, at its $\mathrm{LD}_{50}$ concentration $(100 \mu \mathrm{M})$, caused rapid and reversible currents that were comparable to those evoked by $100 \mu \mathrm{M}$ glutamate (Figure 2, A and B). However, as expected from our previous results, $100 \mu \mathrm{M}$ sulfite (in cystine-free medium) had no effect on basal or postsynaptic currents (Figure 2B). We next tested SSC on somatic NMDA-Rs by examining the effect of the competitive NMDA-R antagonist APV $(50 \mu \mathrm{M})$ and the noncompetitive NMDA-R antagonist MK801 (10 $\mu \mathrm{M})$ on SSC-evoked currents. Indeed, the SSC-evoked current amplitudes were decreased by the competitive antagonist APV $(66.7 \% \pm 7.8 \% ; n=7 ; P<0.01)$ (Figure $2, \mathrm{C}$ and $\mathrm{D})$ and the noncompetitive antagonist MK801 $(48.4 \% \pm 6.7 \% ; n=3 ; P<0.05)$ (Figure 2, C and D). The MK801 effect was not reversible after washout (Figure 2C), which is consistent with the very slow "offrate" of this open-channel blocker (27). Most notably, the residual current could be almost completely attenuated when NMDA-Rs and AMPA-type receptors were blocked simultaneously (remaining current: $7.06 \% \pm 5.00 \%, n=5, P<0.05$ ) (Figure 2, $\mathrm{E}$ and $\mathrm{F}$ ), indicating that SSC acts as an agonist of both types of ionotropic glutamate receptors in neurons.

Multiple studies have reported that excessive depolarization of the neuronal membrane potential due to stimulation of NMDA-R impairs calcium homeostasis, which can trigger multiple signaling cascades that lead to cell death (28-30). We further characterized NMDA-R activation by monitoring intracellular $\mathrm{Ca}^{2+}$ levels, which rise upon receptor stimulation. We used ratiometric $\mathrm{Ca}^{2+}$ imaging with fura-2 to compare cytosolic $\mathrm{Ca}^{2+}$ dynamics in cultured cortical neurons that were induced by acute extracellular application 
A<smiles>N[C@@H](C=O)CCC(=O)O</smiles>
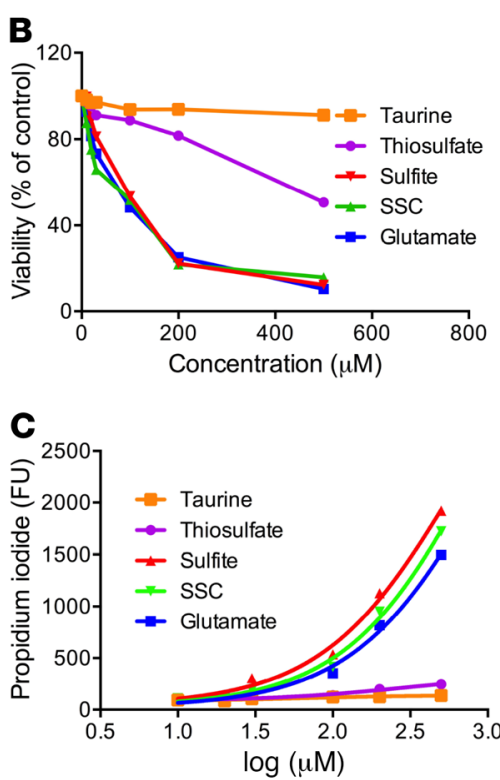

D

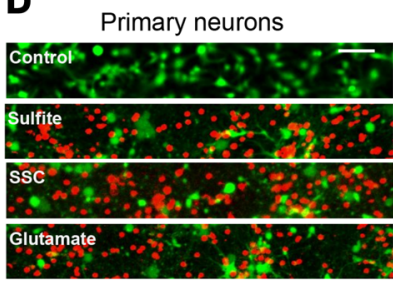

HEK293

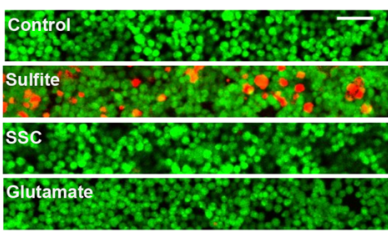

$\mathrm{LD}_{50}(\mu \mathrm{M})$

E

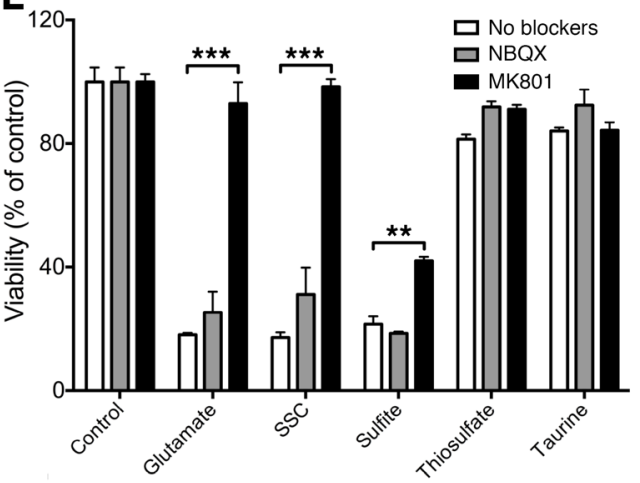

$\mathbf{F}$
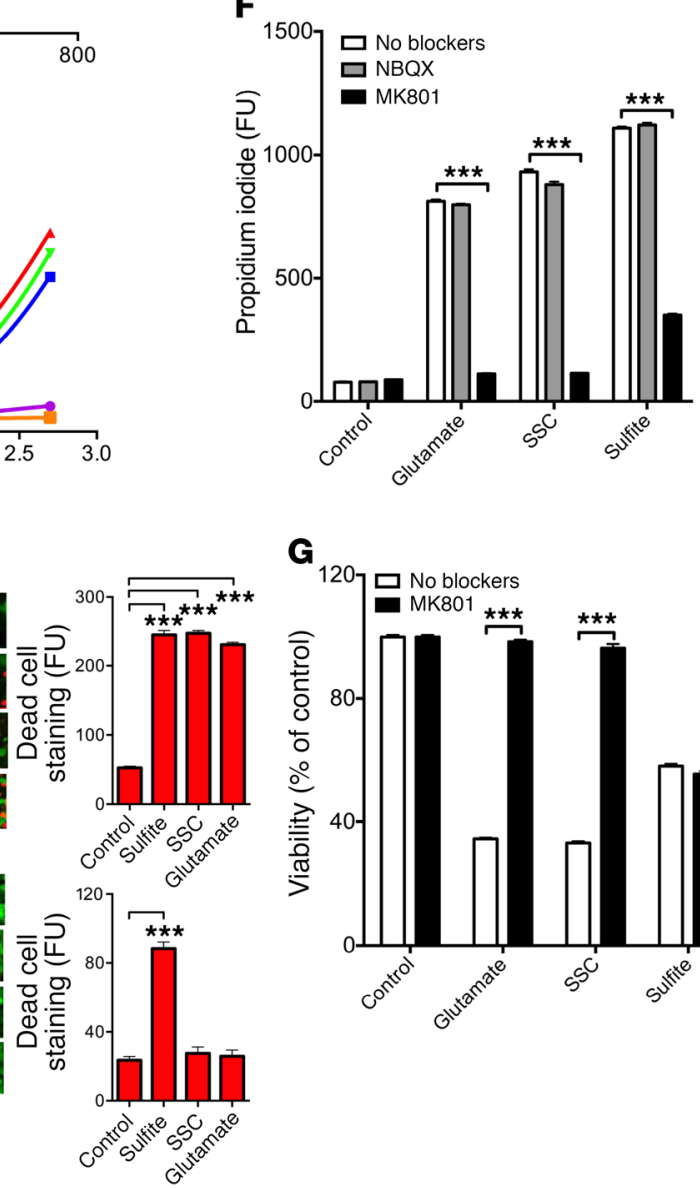

G

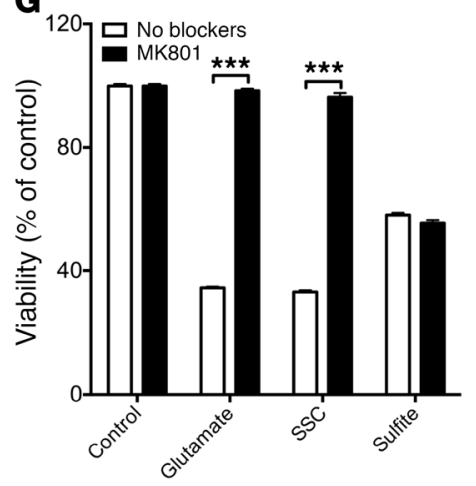

Figure 1. Sulfite toxicity in neuronal tissue is mediated by SSC activation of the NMDA-R. (A) Chemical structure of glutamate and SSC. (B and C) Dosedependent toxicity of taurine, thiosulfate, sulfite, SSC, and glutamate in cortical neurons after a 12-hour incubation in culture medium, as assessed by MTT assay $(n=3)$ (B) or propidium iodide staining $(n=9)(C)$. Cultures were treated with either vehicle (control) or 10, 20, 50, 100, 200, and 500 $\mu \mathrm{M}$ of each of the investigated metabolites, and the $\mathrm{LD}_{50}$ values are highlighted for each metabolite using the MTT assay. (D) Representative images of live/dead staining under control conditions and in the presence of sulfite, SSC, or glutamate in primary neurons and HEK293 cells. Scale bars: $20 \mu \mathrm{m}$. The quantification of dead cells (red signal) was performed by measuring the fluorescence of ethidium homodimer-1 ( $n=6$ for each condition). (E and $\mathbf{F}$ ) Cell viability of cortical neurons measured by (E) MTT assay for glutamate, SSC, sulfite, thiosulfate, and taurine (each $200 \mu \mathrm{M})(n=3)$ and $(\mathbf{F})$ propidium iodide staining for glutamate, SSC, and sulfite at the same concentration $(n=12)$ in the presence of the NMDA-R blocker MK801 $(1 \mu \mathrm{M})$ and the AMPA-R blocker NBQX (20 $\mu M)$. (C) Cell viability of cortical neurons in ACSF media in the absence and presence of the NMDA-R blocker MK801 for glutamate, sulfite, and SSC $(n=3)$. Data are presented as the mean \pm SEM. ${ }^{* *} P<0.01$ and ${ }^{* * *} P<0.001$, by 2 -Way ANOVA with Dunnett's (D-F) or Sidak's (C) multiple comparisons test. FU, fluorescence units. of glutamate or SSC (Figure 3, A-D). Glutamate and SSC induced indistinguishable cytosolic $\mathrm{Ca}^{2+}$ elevations (Figure 3, B and C), which were inhibited to a similar extent by MK801 (Figure 3D).

SSC decreases inhibitory synaptic input through calpaindependent gephyrin degradation. In the course of excitotoxicity, the $\mathrm{Ca}^{2+}$-dependent protease calpain cleaves a number of synaptic proteins at glutamatergic synapses, leading to synaptic loss and eventually neuronal cell death (31). Given that gephyrin, the major regulatory protein at inhibitory postsynapses (32), is also a calpain substrate, we tested whether SSC-mediated $\mathrm{Ca}^{2+}$ influx leads to calpaindependent cleavage of gephyrin (Figure 4) and loss of GABAergic synapses. The well-established calpain-dependent cleavage of spectrin, the major component of the cytoskeletal scaffolding network implicated in brain injuries (33-35), was used as a control. Indeed, SSC application $(100 \mu \mathrm{M})$ to hippocampal neurons induced a time-dependent cleavage of both gephyrin and spectrin. Cleavage of both proteins was prevented in the presence of MK801 or a calpain-specific inhibitor (MDL-28170 or calpain inhibitor III) (Figure 4, A-C). In contrast, the excitatory postsynaptic density protein 95 (PSD95) was less sensitive to SSC or glutamate treatment, and no significant degradation of PSD95 could be detected (Figure 4, A and D). To assess the downstream impact of gephyrin cleavage on postsynaptic GABA type A receptors $\left(\mathrm{GABA}_{\mathrm{A}} \mathrm{Rs}\right)$, which are clustered by gephyrin, we monitored gephyrin-positive GABAergic sites following incubation of neurons 
A

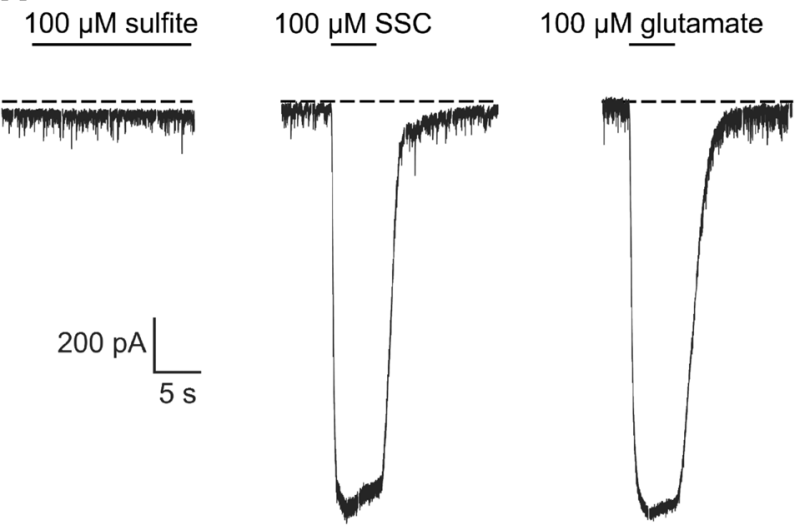

C $\frac{100 \mu \mathrm{M} \text { SSC }}{50 \mu \mathrm{M} \mathrm{APV}}$

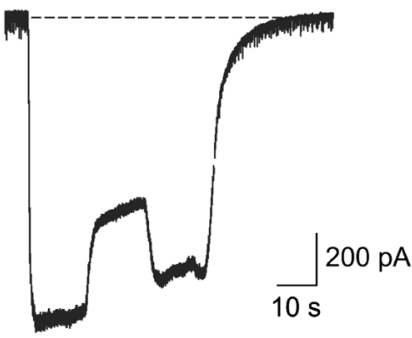

E

$100 \mu \mathrm{M}$ SSC

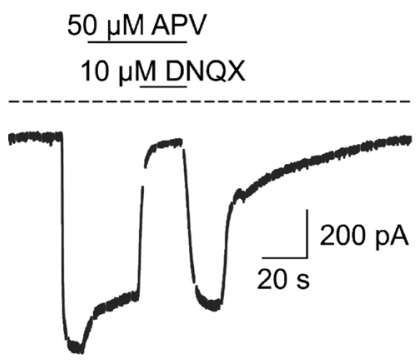

$\mathbf{F}$

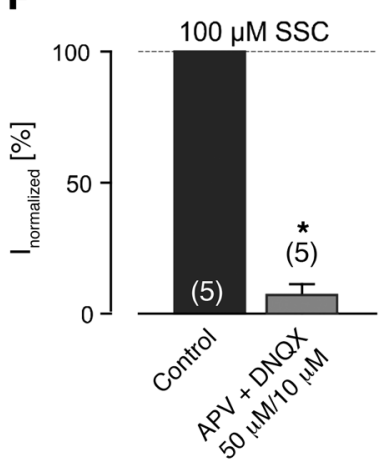

B

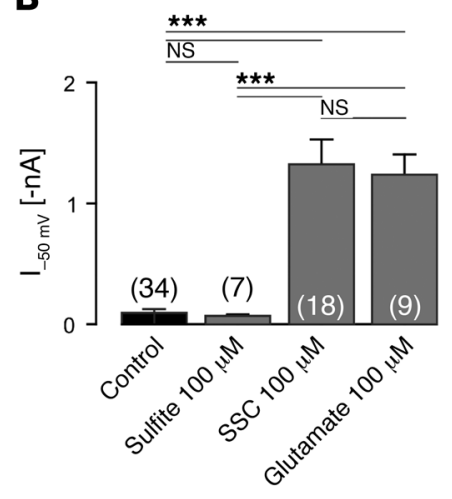

D

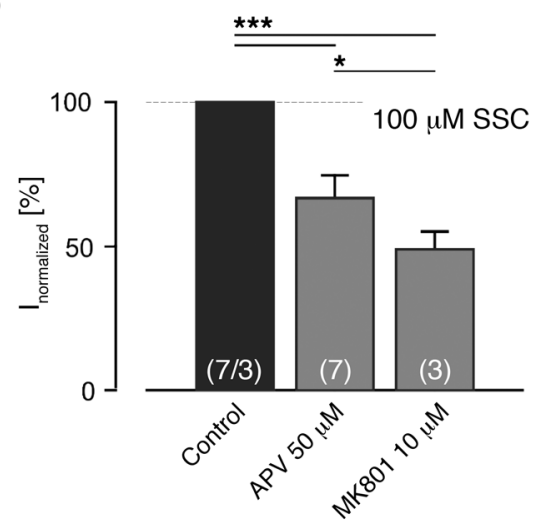

Figure 2. SSC, not sulfite, evokes somatic membrane currents and depolarizes the neuronal membrane potential. (A) Current traces of hippocampal neurons at $-50 \mathrm{mV}$ in the presence of $0.3 \mu \mathrm{M}$ TTX and $100 \mu \mathrm{M}$ sulfite, SSC, or glutamate. (B) Quantification of current amplitudes under basal conditions and in the presence of $100 \mu \mathrm{M}$ sulfite, $100 \mu \mathrm{M}$ SSC, or $100 \mu$ M glutamate. (C) NMDA-R antagonists markedly decreased SSC-evoked current amplitudes in hippocampal neurons. (D) Quantification of the percentage of inhibition of tonic SSC-evoked currents by NMDA-R antagonists. Current amplitudes in the presence of $10 \mu \mathrm{M}$ MK801 or $50 \mu \mathrm{M}$ APV were normalized to SSC-induced current peaks. (E) Application of both APV and DNQX almost completely blocked SSC-elicited currents. (F) Quantification of the additive effect of NMDA-R and AMPA-R blocker on current amplitudes. Data are presented as the mean \pm SEM. Numbers in parentheses in $\mathbf{B}$, $\mathbf{D}$, and $\mathbf{F}$ indicate the number of recorded neurons. ${ }^{*} P<0.05$ and ${ }^{* *} P<0.001$, by 1 -way ANOVA with Tukey's multiple comparisons test. with SSC or glutamate $(100 \mu \mathrm{M})$ (Figure $4 \mathrm{E})$. Treatment with SSC or glutamate significantly decreased the gephyrin-positive cluster density (Figure $4 \mathrm{~F}$ ) and size (Figure 4, G and H), both of which were unchanged in the presence of MK801 or calpain inhibitor.

Since gephyrin is crucial for the function of inhibitory GABAergic synapses, these results indicate that cleavage of gephyrin upon SSC treatment could impair GABAergic inhibition and therefore augment NMDA-R-mediated excitotoxicity. To probe the contribution of each component of this pathway on SSC-mediated toxicity, we tested the NMDA-R blocker MK801, the $\mathrm{Ca}^{2+}$ scavenger BAPTA-AM, and the calpain inhibitor III (Figure 4, I and J) and found that they were individually equally effective in preventing SSC and glutamate toxicity (Figure 4, I and J), thus demonstrating an important contribution of the SSC-mediated NMDA-R opening/calcium influx/calpain activation cascade in the pathomechanism of MoCD. In accordance with this, we did not observe any cellular SSC uptake in cortical neurons under the culture conditions used, which further supports the idea that SSC exerts its neurotoxicity from the extracellular side (Supplemental Figure 3).

Pharmacological induction of MoCD in mice confirms SSC neurotoxicity. To study the impact of SSC-mediated toxicity in vivo, we used a pharmacologically induced model of MoCD. Animals exposed to tungstate experience a dose-dependent replacement of molybdenum by tungsten during Moco synthesis, leading to the inactivation of all Moco-dependent enzymes (36, 37). Fourweek-old mice exposed to sodium tungstate at doses of up to 1,000 ppm showed a dose-dependent decrease in liver SO activity ( $40 \%$ median decrease in 1,000 ppm versus control), while tungstate doses of $8,000 \mathrm{ppm}$ led to a median decrease of $90 \%$ in SO activity when compared with controls (Figure 5A). This group of animals developed typical MoCD symptoms within 3 weeks, including weight loss (Figure $5 \mathrm{~B}$ ), spontaneous twitching, hunched-back posture, and reduced mobility, all of which 

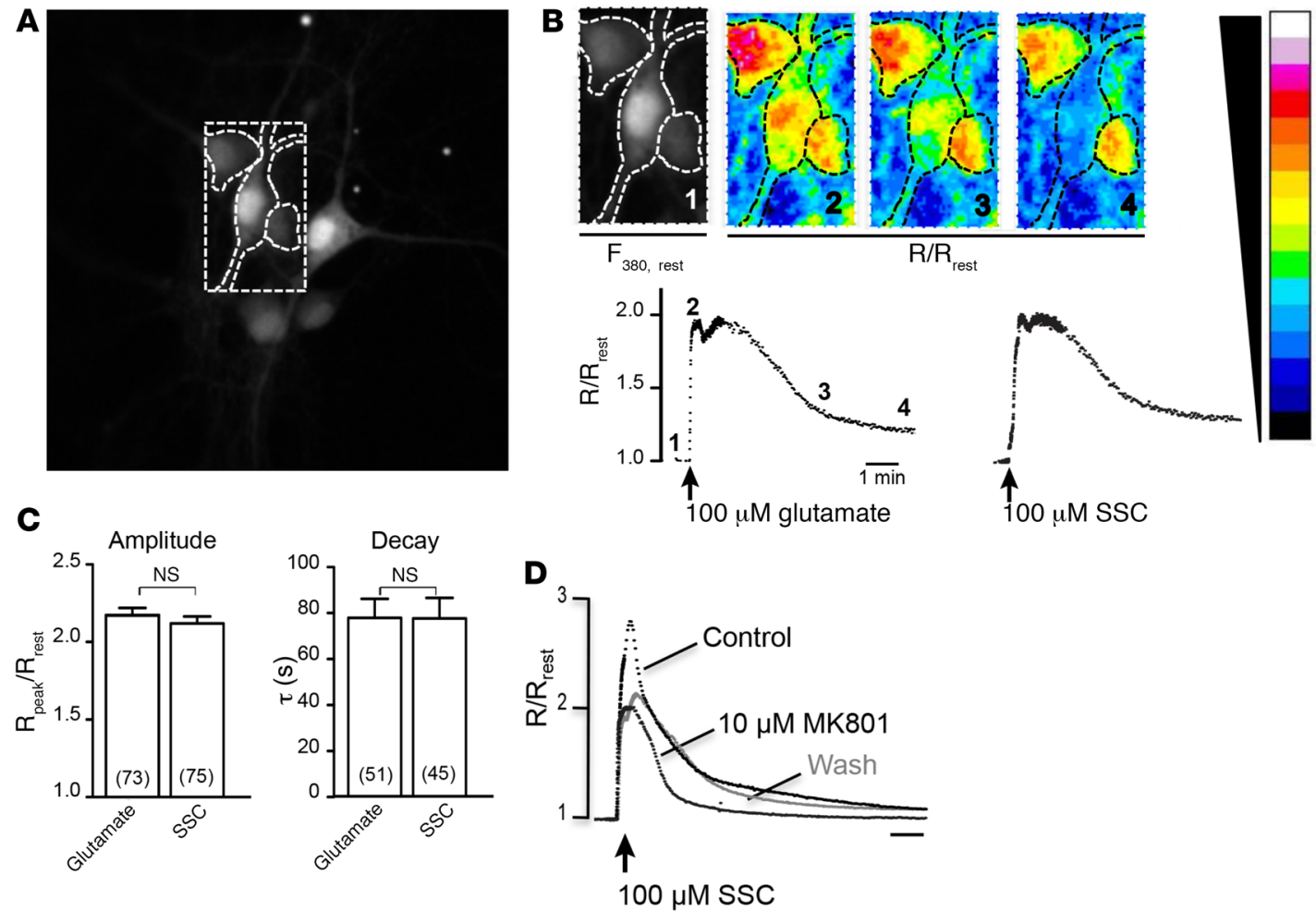

Figure 3. SSC, like glutamate, induces calcium influx (32. (A) Fluorescence image $\left(F_{380}\right)$ of fura-2 AM-loaded cortical neurons. (B) Glutamate and SSC induced a similar $\mathrm{Ca}^{2+}$ influx. (B) Intracellular $\mathrm{Ca}^{2+}$ dynamics of a cortical neuron in response to a 10 -second bath application of $100 \mu \mathrm{M}$ glutamate (left trace) and $100 \mu \mathrm{M} \mathrm{SSC} \mathrm{(right} \mathrm{trace).} \mathrm{The} \mathrm{images} \mathrm{show} \mathrm{the} \mathrm{framed} \mathrm{region} \mathrm{of} \mathbf{A}$ at higher magnification and demonstrate the glutamate-induced increase in cytosolic $\mathrm{Ca}^{2+}$ concentration. The numbers indicate the time points of the traces when the images were taken. (C) Maximal amplitude and decay time constant of the glutamate- and SSC-induced rise in cytosolic Ca ${ }^{2+}$. The numbers in parentheses indicate the number of recorded neurons. (D) SSC-induced $\mathrm{Ca}^{2+}$ dynamics of a cortical neuron before, during, and after application of the NMDA-R blocker MK801. Data are presented as the mean \pm SEM. Data were analyzed using a 2-tailed, unpaired Student's $t$ test.

became increasingly severe after 4 weeks of treatment (Supplemental Videos 1 and 2).

SSC levels in urine and brain extracts were elevated in tungsten-treated animals, with considerable variation in urine levels (Figure 5, C and D), which correlated inversely with the residual activity of SO in each animal (Supplemental Figure 4, A and B). We analyzed a second cohort of mice and found similar SSC values in brain extracts, with a combined median of $72 \mathrm{nmol} / \mathrm{mg}$ protein for both cohorts (Fi gure 5D). We also found a moderate 2-fold increase in SSC levels in liver as compared with levels in controls, with a median of $1 \mathrm{nmol} / \mathrm{mg}$ protein, thus leading to a 70 -fold increase in SSC levels in brain as compared with those in liver (Figure 5D). To our knowledge, this is the first report to demonstrate that SSC indeed accumulates dramatically in the brains of MoCD mice.

Consistent with our results in cultured neurons, gephyrin and spectrin degradation was observed in brain extracts from tungsten-treated animals (Figure 5E), while PSD95 levels were not altered (Figure 5F). In contrast, we observed no degradation of gephyrin or spectrin in liver, where both proteins are also expressed (Figure 5E), suggesting that SSC-mediated calpain cleavage of gephyrin and spectrin is exclusive to the brain and involves NMDA-R signaling. To further investigate brain injuries in tungsten-treated mice, we analyzed different brain sections from control and tungsten-treated animals (Figure 5G). In con- trast to Mocs1-KO mice (20), we observed neuronal cell death in tungsten-treated mice using Nissl staining, which revealed strong abnormalities in cerebral cortex and the CA1 region of the hippocampus of tungsten-treated animals (Figure 5G, arrows). Thus, our MoCD mice developed symptoms similar to those in patients, recapitulating the results obtained with cultured neurons and demonstrating the potential of SSC to act on glutamatergic pathways to induce neurodegeneration in MoCD.

Memantine improves the performance of MoCD mice. To evaluate the pharmacological potential for the use of NMDA-R antagonists in the treatment of MoCD, we tested memantine, an NMDA-R blocker used in the treatment of Alzheimer's disease. In contrast to MK801, memantine is a low-affinity, uncompetitive antagonist able to inhibit prolonged $\mathrm{Ca}^{2+}$ influx, particularly from extrasynaptic receptors, without impairing receptor function at synapses. We first tested memantine in vitro and confirmed its potential to rescue SSC-mediated neuronal cell death (Figure 6A) and gephyrin cleavage (Supplemental Figure 5), as was observed with MK801.

In healthy humans, SSC is normally not present in CSF at any detectable level $(<1 \mu \mathrm{mol} / \mathrm{l})$ (38), while in SOD, SSC levels of 19 $\mu \mathrm{mol} / \mathrm{l}$ were reported (38). In addition to our previous studies, we examined the potential of low SSC levels to induce cell death over a prolonged incubation period of 5 days in primary neurons. We 
$\mathbf{A}$
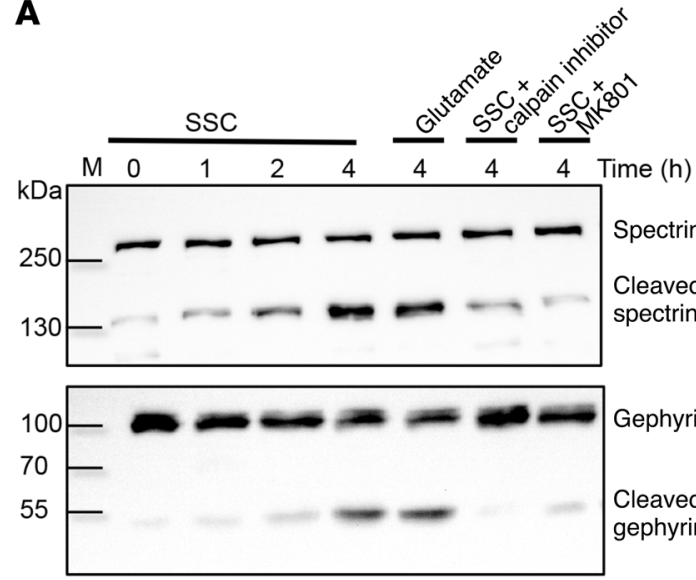

Gephyrin

Cleaved gephyrin

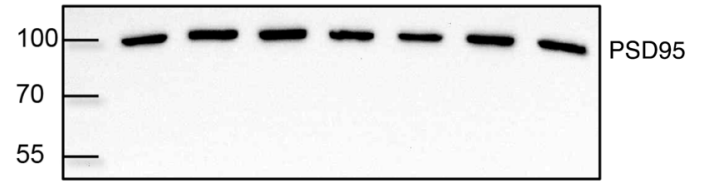

$55 \square-\infty-2-$-Tubulin

D

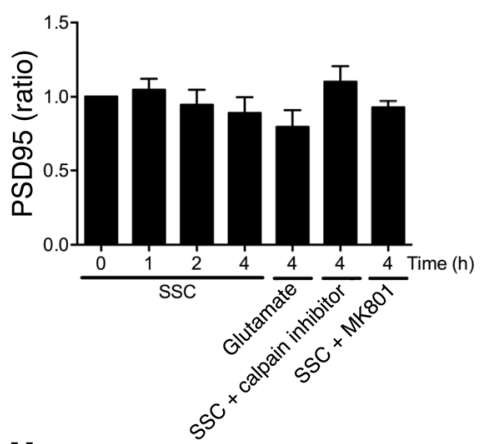

H

E
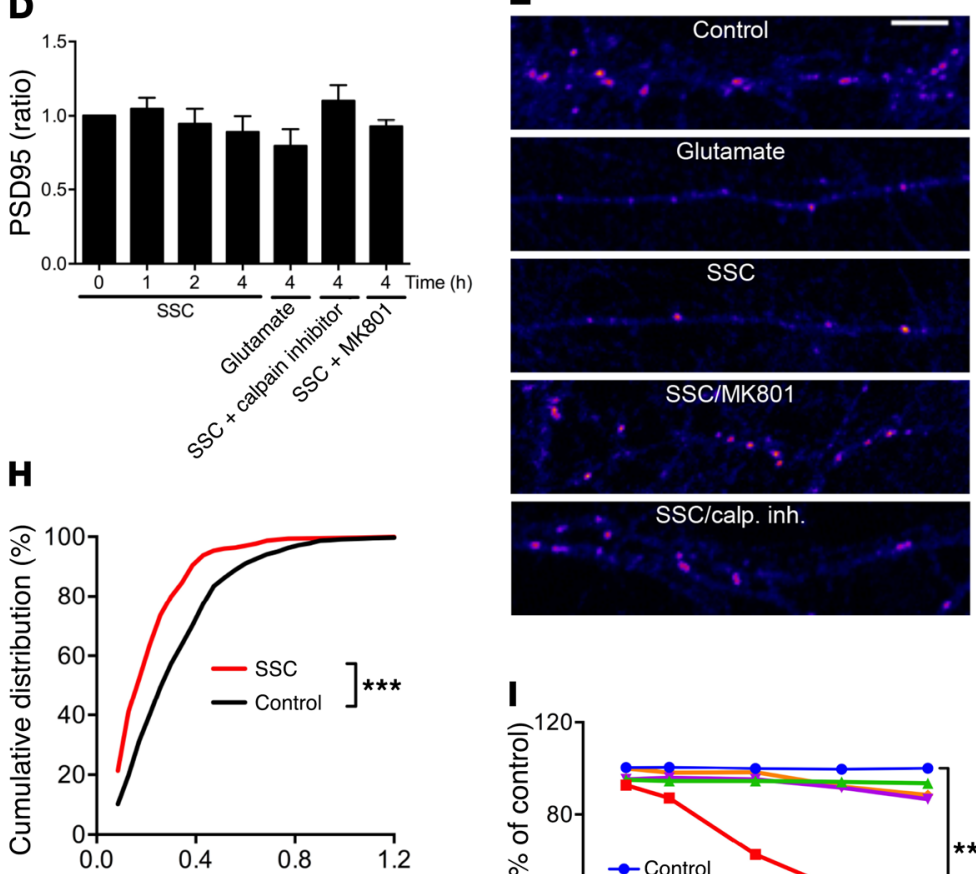

Gephyrin cluster size $\left(\mu \mathrm{m}^{2}\right)$

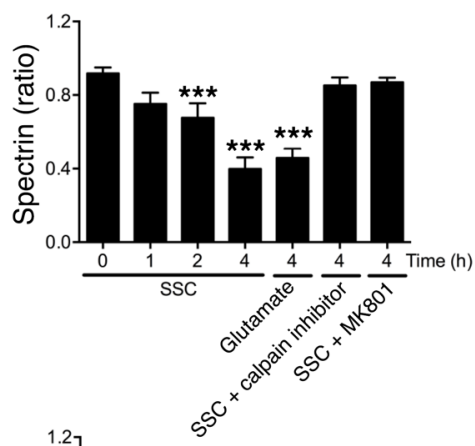

B

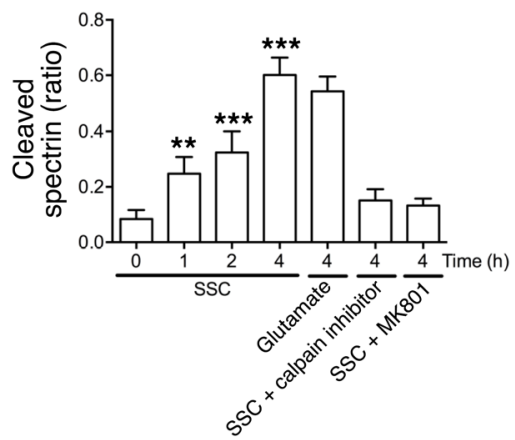

C

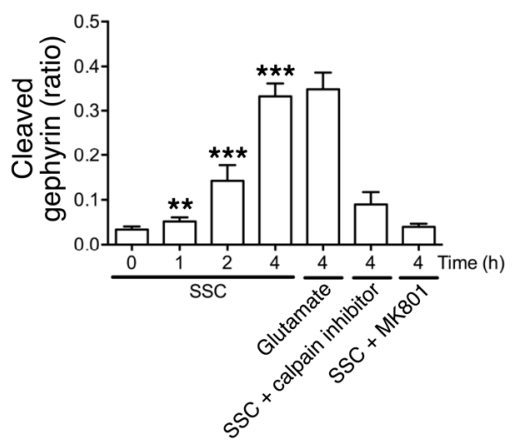

$\mathbf{F}$

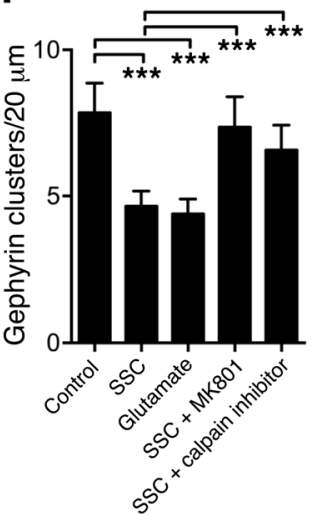

G

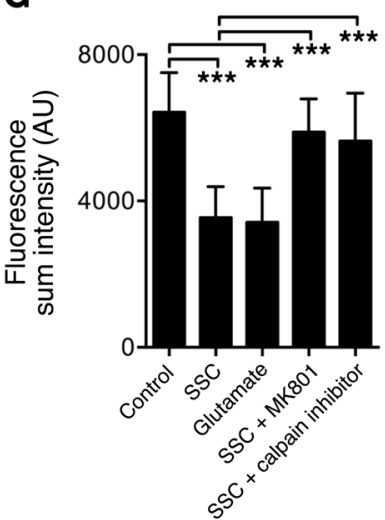

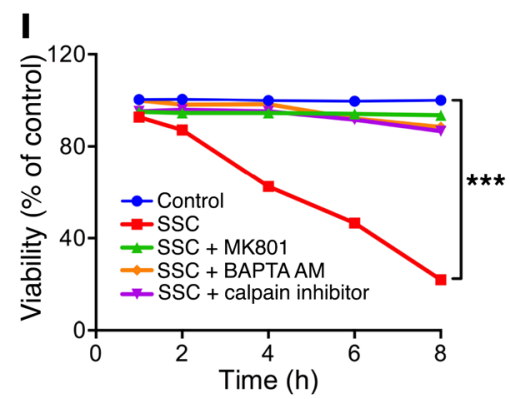

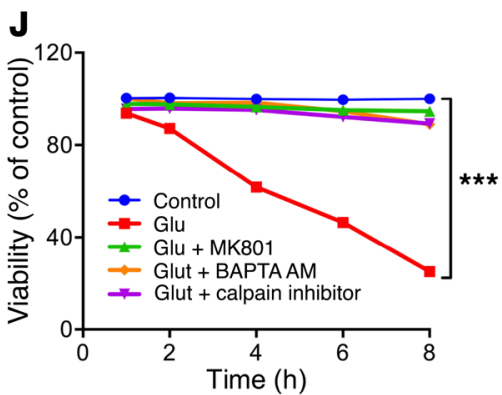

Figure 4. SSC-mediated calcium influx activates calpain and leads to gephyrin degradation. (A) Representative Western blots of spectrin, gephyrin, and PSD95 in hippocampal neurons treated with glutamate $(100 \mu \mathrm{M})$, SSC alone $(100 \mu \mathrm{M})$, SSC plus calpain inhibitor $(10 \mu \mathrm{M})$, or SSC plus MK801 $(1 \mu \mathrm{M})$ at different time points. M, molecular weight marker. Quantification of band intensities of full-length and cleaved spectrin (B), gephyrin (C), and full-length PSD95 (D). At least 5 Western blots per condition from 3 independent neuronal preparations were used. (E) Representative images of dendrites immunostained for gephyrin after a 2-hour incubation with SSC, glutamate, or the indicated inhibitors. Scale bar: $5 \mu \mathrm{m}$ (F) Gephyrin cluster density, (G) total fluorescence intensity of clusters, and (H) cumulative distribution of gephyrin cluster size in dendrites after treatment of neurons as in $\mathbf{E}$ (number of dendritic segments analyzed: control = 59; SSC = 67; glutamate $=71$; SSC plus calpain inhibitor $=39$; SSC plus MK801 = 54, from 2 independent cultures). (I and J) Time-dependent toxicity studies were conducted in cortical neurons for SSC (I) and glutamate (J) (each $200 \mu \mathrm{M})$ in the absence and presence of the NMDA-R blocker MK801 (1 $\mu$ M), the calcium scavenger BAPTA-AM $(10 \mu \mathrm{M})$, and calpain 1 inhibitor $(10 \mu \mathrm{M})$. Data are presented as the mean \pm SEM. ${ }^{* *} P<0.01$ and ${ }^{* *} P<0.001$, by 2-way ANOVA with Dunnett's (B-D and $\left.\mathbf{H}-\mathrm{J}\right)$ or Tukey's (F and $\mathbf{G})$ multiple comparisons test. 
found that SSC levels of 1 to $10 \mu \mathrm{M}$ were able to induce neuronal cell death, which was reversed equally well by both memantine and MK801 treatment (Figure 6B). Furthermore, we observed a dose-dependent depolarization effect of SSC on membrane potential in the current-clamp recording condition $(1 \mu \mathrm{M}$ SSC, $2.22 \pm$ $0.76 \mathrm{mV}, n=3 ; 10 \mu \mathrm{M}$ SSC, $29.02 \pm 2.00 \mathrm{mV}, n=14 ;{ }^{* * *} \mathrm{P}<0.001$; $100 \mu$ M SSC, $43.62 \pm 0.92 \mathrm{mV}, n=16 ;{ }^{* * *} P<0.001$ ) (Figure 6C). These results demonstrate that even low concentrations of SSC can induce strong depolarizations of the neuronal membrane potential and, hence, that SSC is a strong excitatory neurotransmitter.

Finally, we tested the impact of memantine on a separate cohort of tungstate-treated mice, both to validate the molecular pathway we propose and to demonstrate the potential of memantine as a new therapeutic for MoCD. Because of the severity of the disease observed in the first cohort of mice (molybdenumdeficient diet and tungsten supplementation), which resulted in death after 4 weeks of treatment, the mice were fed a normal diet (normal molybdenum content), and their drinking water was supplemented with the same effective tungsten dose $(8,000 \mathrm{ppm})$ used previously, which allowed the experiment to be conducted for more than 5 weeks. Mice received i.p. injections of memantine twice a week, and the mice were assessed weekly for body weight and motoric performance using the rotarod instrument.

To exclude tungsten enrichment in neuronal tissue, which may indicate direct tungsten neurotoxicity in our pharmacological mouse model, we measured tungsten levels in the livers, kidneys, and brains of the treated mice using inductively coupled plasma mass spectrometry (ICP-MS) at the end of the study. We found that tungsten levels were elevated in the liver and kidneys of the mice (organs with the highest capacity for Moco synthesis and sulfite oxidation [ref. 22]), however, none of the mice had elevated tungsten levels in the brain. We also found that tungsten levels were not altered by memantine treatment. These findings indicate that the observed effects of tungsten intoxication are due to the loss of SO activity in the periphery and not to direct neurotoxicity of tungsten (Supplemental Figure 6A). Additionally, in vitro data showed that neuronal cell lines are less susceptible to tungsten toxicity than are HEK293 cells (Supplemental Figure 6B). Consistent with our previous results (Figure 5B), tungsten treatment induced progressive weight loss in mice (Figure 6D) and a significant decrease in rotarod motoric performance (Figure 6E) after 4 and 5 weeks of treatment. More important, memantine treatment completely rescued tungsten-induced weight loss and rotarod impairment after 4 and 5 weeks of treatment (Figure 6, D and E). In accordance with our in vitro data, memantine was also able to decrease gephyrin degradation in both control and tungsten-treated animals, while no degradation was observed for PSD95 (Figure 6F), thus demonstrating the therapeutic potential of NMDA-R antagonists in MoCD.

In summary, we demonstrated the following sequence of events accounting for neuronal cell death in MoCD: (a) SOD induces a systemic increase in sulfite, (b) sulfite accumulation results in SSC formation within the periphery as well as the brain following the reaction of excess sulfite with cystine, and (c) SSC induces excitotoxicity via NMDA-R-dependent calcium signaling and calpain activation (Figure 6G).

\section{Discussion}

Although MoCD results in the deficiency of 4 enzymes in humans, SOD is generally accepted as the major cause of brain damage in MoCD (2). Little is known about the metabolites involved in neuronal cell death in MoCD; the purpose of this study was to determine the underlying pathomechanism of the disease, in particular, the potential involvement of the S-metabolites. We identified and confirmed sulfite and SSC as the main mediators of neuronal toxicity. Sulfite toxicity has been investigated in the past and was proposed as the major trigger of neuronal cell death in $\operatorname{MoCD}(4,20)$. Sulfite increases oxidative stress and reduces ATP synthesis in mitochondria respiring on glutamate (26). Sulfite is a strong reductant and reacts with disulfide bonds in proteins (26) and cystine, which we demonstrated in vitro in this study. High levels of excreted SSC and sulfite, together with cystine depletion, are characteristic of human patients, supporting the hypothesis that an excess of sulfite crosses the blood-brain barrier to cause neurodegeneration.

In addition to sulfite, our data identified SSC as the major trigger of neuronal cell death in MoCD. SSC-mediated activation of NMDA-R and AMPA-R led to dose-dependent depolarization of the neuronal membrane potential, which increased neuronal excitability and intracellular calcium levels and triggered the activation of downstream cell death pathways. In particular, we found that the calcium-dependent protease calpain was activated upon exposure of neurons to SSC (and glutamate), which upregulated the degradation of neuronal proteins and cell death. SSC-triggered neurotoxicity could be rescued with an NMDA-R antagonist, calcium scavenger, and calpain inhibitor. The action of SSC on neuronal cells was pharmacologically indistinguishable from that of glutamate. However, the selective enrichment of SSC found in the brains of tungsten-treated mice compared with that found in livers may indicate the lack of an efficient SSC clearance from the brain, which would result in a prolonged overstimulation of ionotropic glutamate receptors and enhanced excitotoxicity. It should be noted that the SSC levels determined here were measured in total brain extracts, and future studies are required to determine the reference values for extracellular/CSF levels of SSC, which would be more informative and predictive of neuronal damage. Because SSC toxicity was restricted to neuronal cells in contrast to sulfite, and given the severe neurological symptoms characteristic of MoCD, we argue that SSC is the principal mediator of neurotoxicity in MoCD.

Gephyrin plays a crucial role in the clustering and plasticity of inhibitory synapses $(32,39)$, and either genomic deletions or miss-splicing within the GPHN gene has been linked to neurological disorders such as epilepsy $(40,41)$, schizophrenia, and hyperekplexia $(42,43)$. Furthermore, gephyrin is also crucial for Moco synthesis, as it catalyzes the 2-step metal insertion reaction (44). Consistent with this, Gphn-KO mice exhibit a neurological phenotype more severe than that of MoCD; indeed, homozygous-KO mice die within the first day of life (45). Our finding of an SSCmediated reduction in GABAergic transmission, accompanied by pronounced depolarization of the neuronal membrane potential, suggests that the increased neuronal excitability in neuronal circuits of MoCD patients contributes to the severity of their seizures, followed by rapidly progressing loss of brain tissue (Figure 
A

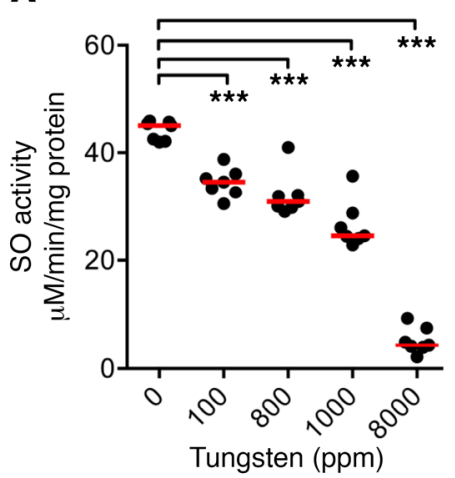

B

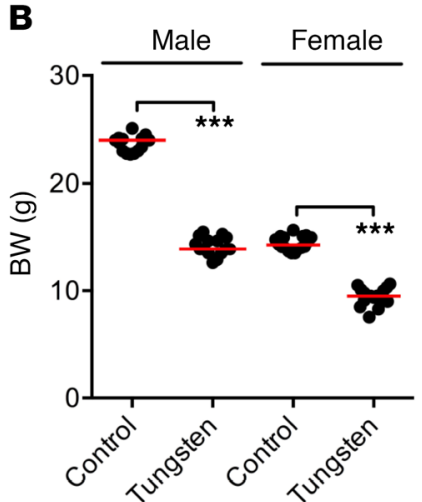

C

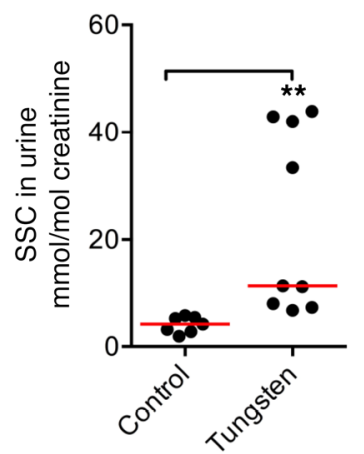

D

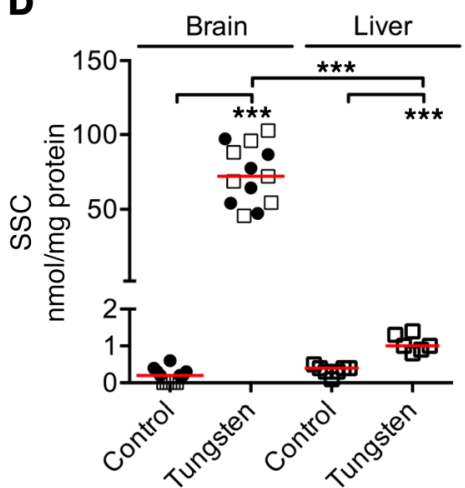

E
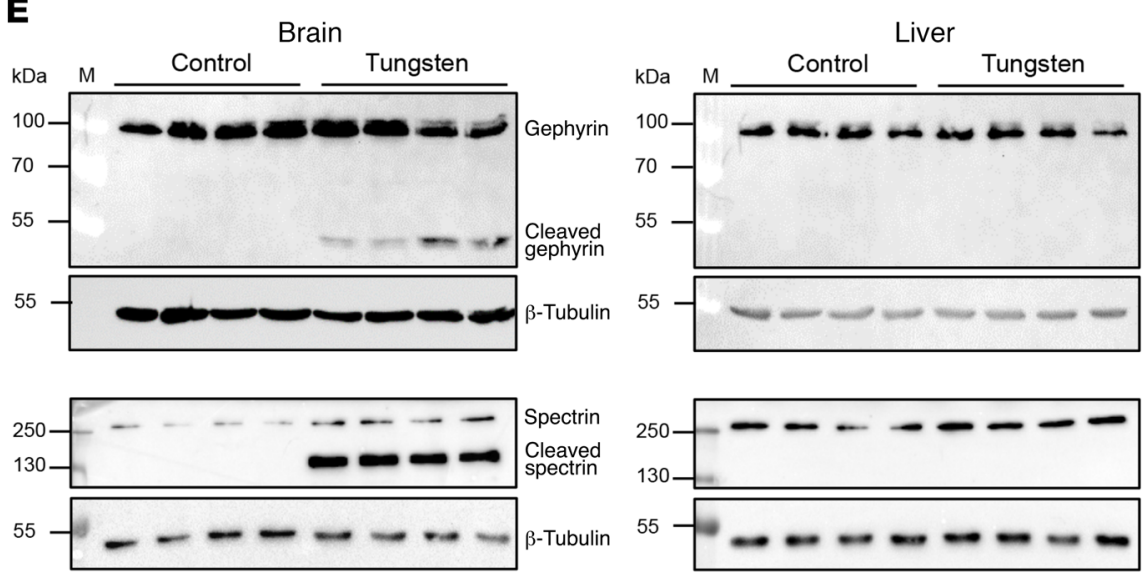

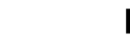

$\mathbf{F}$

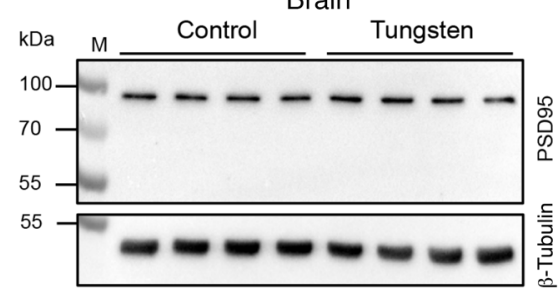

G
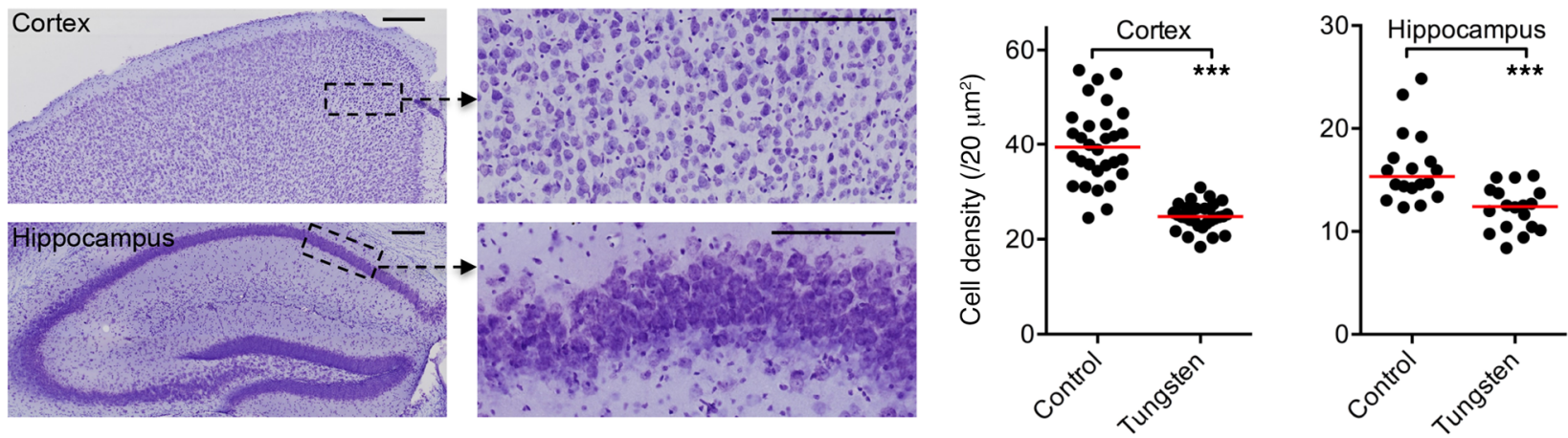

Cortex

Tungsten
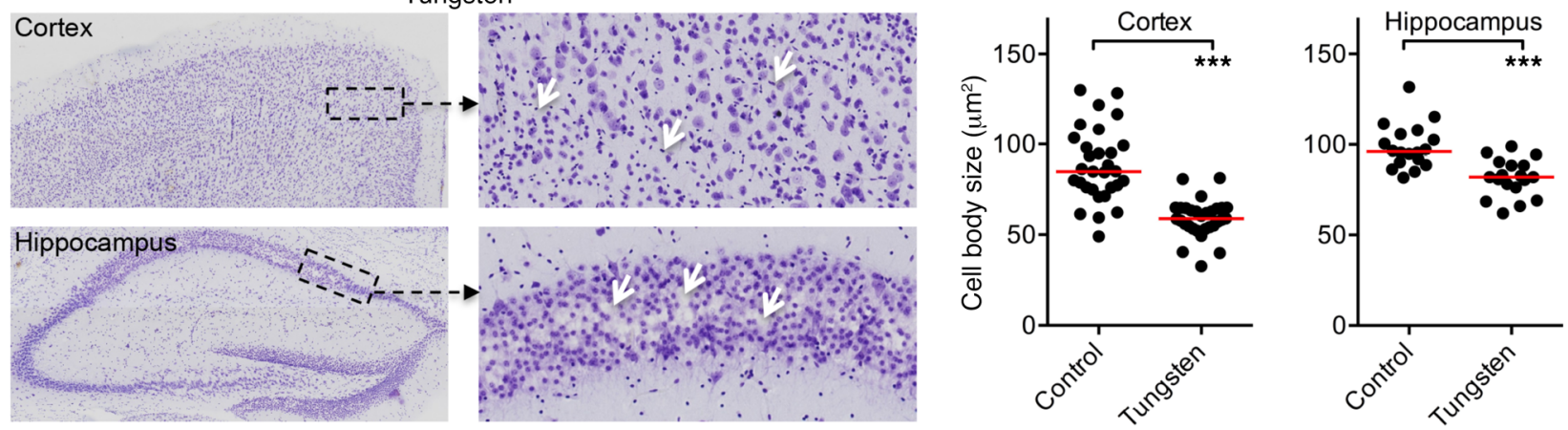
Figure 5. Induction of MoCD in mice prompts SSC formation and neuronal cell death. (A) Liver SO activity in mice after 4 weeks of treatment $(n=7)$ group). (B) Assessment of body weights ( $n=32$ mice/treatment group) of male and female mice. (C) SSC accumulation in urine ( $n=7$ control mice, $n=9$ tungsten-treated mice). (D) Normalized SSC levels in extracts of brain (black circles and white squares represent 2 individual mice cohorts; $n=12$ control, $n=13 \mathrm{SSC}$ ) and liver ( $n=7$ control, $n=6 \mathrm{SSC}$ ). (E) Calculated SSC concentration in brain extracts from $\mathbf{D}(n=12$ control, $n=13 \mathrm{SSC}$ ). (E and F) Immunoblots showing the expression of gephyrin and spectrin in brain and liver extracts $(\mathbf{E})$ and the expression of PSD95 in brain extracts $(\mathbf{F})$ from control and tungsten-treated mice ( $n=4 /$ group). (G) Nissl-stained images of brain sections from control and tungsten-treated mice, with representative sections of cortical and hippocampal regions and quantification of neuronal density and size in cortex (layers 1 and 2 ) and hippocampal CA1 regions ( $n=32$ cortex, $n=18$ CA1, derived from 3 mice/group). Scale bar: $200 \mu \mathrm{m}$. Data are presented as the mean \pm the SEM. Red lines indicate the median value. ${ }^{* *} P<0.01$ and ${ }^{* * *} P<0.001$, by 2 -way ANOVA with Dunnett's multiple comparisons test (A), 2-tailed, unpaired Student's $t$ test (B and C), 1-way ANOVA with Tukey's multiple comparisons test (D), or 2-tailed, paired Student's $t$ test (G).

6C). The sequence of events identified in this study is supported by previous findings of maladaptive changes in gephyrin function as a pathogenic factor in epilepsy $(40,41,46,47)$.

We previously characterized the genetic mouse model of human MoCD (20). Mocs1-KO mice display symptoms resembling human MoCD, and these mice were successfully used for the development of the first treatment of human MoCD (20). However, Mocs1-deficient mice die within the first 11 days after birth, without visible brain injuries such as atrophy, cysts, or loss of cortical tissue (20). The lack of overt neuropathological changes, probably due to the mouse's short lifespan, limits the use of this genetic mouse model to investigate the process of neurodegeneration in MoCD. We therefore used a tungstate treatment model of MoCD, which has been successfully used in rats to induce SOD (37). Pharmacological induction of MoCD in older mice allows for prolonged disease development and a more detailed observation of brain pathologies. Tungsten-treated mice developed symptoms similar to those observed in human patients, including weight loss, motoric weakness, and neuronal cell death. In addition, we showed that brain extracts from tungsten-treated mice had SSC accumulation and gephyrin and spectrin cleavage and that PSD95 levels were unaffected. We showed a specific accumulation of SSC in brain tissue, which may suggest prolonged neuronal excitotoxicity and explain the rapid and progressive neuronal cell death observed in human patients. Although, CSF levels of SSC would be more informative of the severity of the pathological condition, it should be noted that SSC is close to detection limits under normal conditions and that SSC only accumulates under disease conditions such as MoCD and SOD. This is consistent with the accumulation of SSC in biological fluids such as urine and plasma, which is used as a disease-predicting and treatment-monitoring biomarker $(10,17,38)$.

In summary, our study provides both in vitro and in vivo evidence that SSC is a major neurotoxic agent in MoCD. SSC is generated by the reaction of sulfite with cystine and was found to specifically accumulate in the brain. Suppression of either SSC-mediated NMDA-R activation or downstream signaling events is sufficient to rescue neuronal survival. We found that
SSC/NMDA-R/calcium signaling resulted in calpain-dependent cleavage of the postsynaptic scaffolding protein gephyrin, causing a destabilization of GABAergic synapses. GABAergic neurotransmission is a common target for anticonvulsive therapies, and patients with MoCD are often treated with phenobarbital and midazolam $(10,21)$. Moreover, our study reveals a therapeutic strategy for the treatment of MoCD by targeting NMDA-Rs, e.g., with memantine, which has been shown to be safe and well tolerated (48). Given our recent clinical findings on MoCD type A patients who were treated with cPMP (19), early diagnosis and a better understanding of the underlying disease mechanism are crucial for treatment success. Therefore, any treatment aimed at delaying neuronal cell death triggered by SSC-mediated NMDA-R activation will increase the time window of treatment for patients who can benefit from cPMP treatment. Our results strongly suggest that targeting the NMDA-R by antagonists would be beneficial in preventing SSC-induced brain damage. This approach is supported by a previous report of a patient with MoCD who had a promising outcome (49). Furthermore, the current classical anticonvulsive treatment for symptomatic MoCD patients is characterized by a progressive lack of treatment response, which, in light of the findings in this study, could be explained by the reduction in GABAergic synapses. In summary, we believe this study translates a mechanistic understanding into a new concept for disease management and potential new therapies for a devastating excitotoxic disease.

\section{Methods}

Cell culture and toxicity studies. Human embryonic kidney cells (HEK293) were cultivated in DMEM supplemented with 10\% FCS and $2 \mathrm{mM} \mathrm{L}$-glutamine at $37^{\circ} \mathrm{C}$ and $5 \% \mathrm{CO}_{2}$. Primary neuron cultures were prepared from hippocampi or cortex of C57BL/6 mice and cultured in neurobasal medium supplemented with B-27, N-2, and L-glutamine. All reagents were supplied by Life Technologies (Thermo Fisher Scientific). Neurons were cultivated at different densities depending on which culture plate was used (30,000/well for 96-well plates, 75,000/well for 24-well plates, and 900,000/well for 6-well plates). For confocal laser-scanning microscopy, neurons and HEK293 cells were cultivated on poly-L-lysine-coated and collagenized coverslips, respectively. Unless otherwise stated, all neuronal studies were conducted after 9 to 10 days in vitro (DIV).

Toxicity studies using the MTT assay were conducted in 96-well plates for 12 hours in the presence of $200 \mu \mathrm{M}$ of each investigated metabolite, unless otherwise stated. For selective neuronal toxicity studies, 2 different receptor blockers were used: MK801 for NMDA-Rs and NBQX for AMPA-Rs, at a final concentration of 1 and $20 \mu \mathrm{M}$, respectively. All amino acids and S-metabolites (sulfite, thiosulfate, taurine and S-sulfocysteine) were purchased from MilliporeSigma, and MDL-28170 (Santa Cruz Biotechnology) was used as a cell-permeable selective inhibitor of calpain 1 . For toxicity experiments in cystine-free medium, neuronal medium was exchanged for an ACSF solution $(124 \mathrm{mM} \mathrm{NaCl}$, $2.5 \mathrm{mM} \mathrm{KCl}, 2.5 \mathrm{mM} \mathrm{CaCl}_{2}, 1.25 \mathrm{mM} \mathrm{KH}_{2} \mathrm{PO}_{4}, 2 \mathrm{mM} \mathrm{MgSO}_{4}, 26 \mathrm{mM}$ $\mathrm{NaHCO}_{3}, 1.3 \mathrm{mM} \mathrm{MgCl}, 10 \mathrm{mM}$ glucose, $4 \mathrm{mM}$ sucrose), and neurons were incubated for 1 hour prior to the start of the experiment.

Cell viability assay. Cell viability studies were conducted using either the MTT assay (Promega) or propidium iodide staining (MilliporeSigma). Cortical neurons (DIV 10) were cultivated in a 

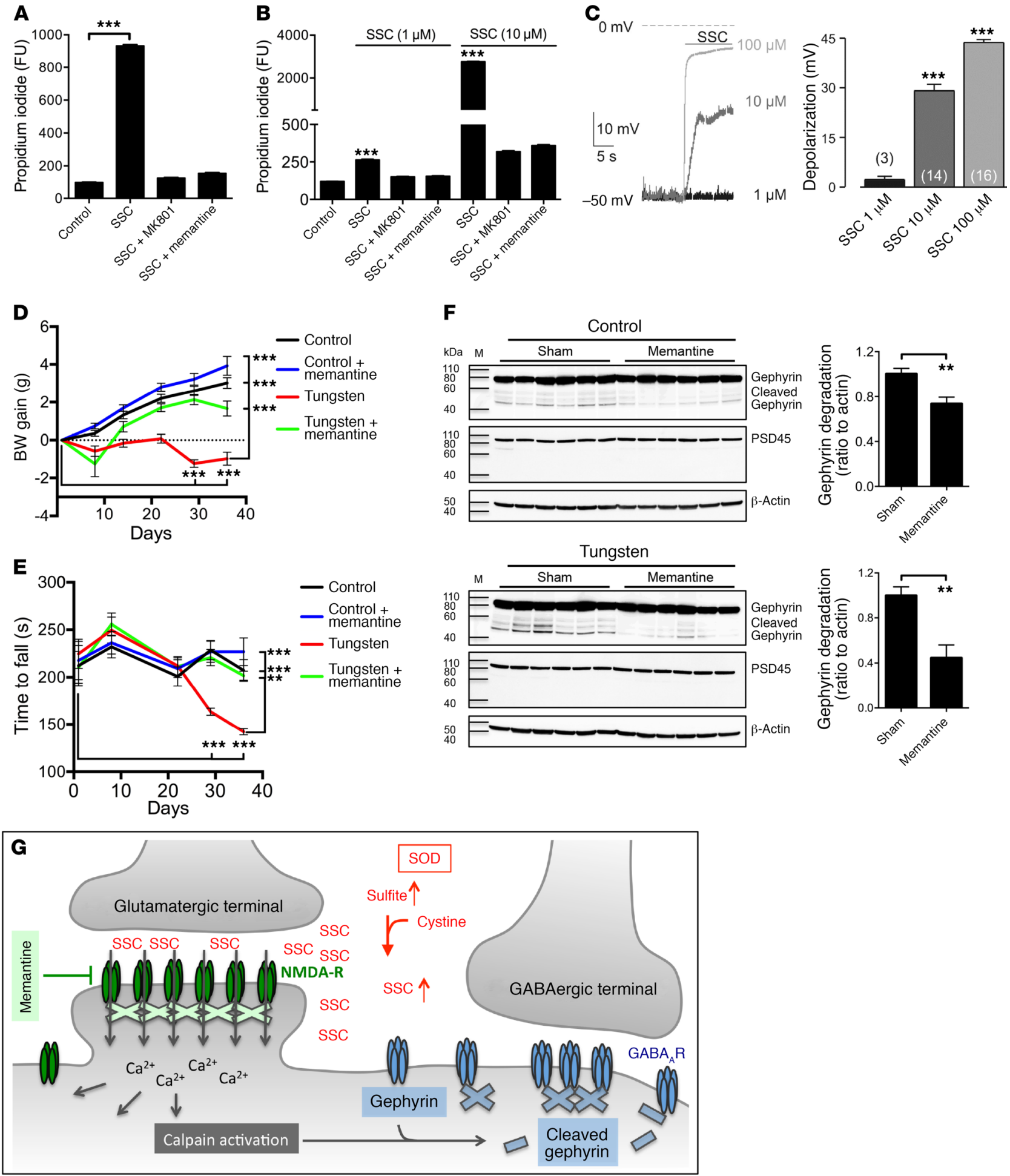

Figure 6. NMDA-R antagonist is beneficial for the treatment of MoCD in mice. (A) Cell viability of cortical neurons with SSC (200 $\mu$ M) was assessed with propidium iodide staining in the absence and presence of the NMDA-R blocker MK801 (1 $\mu \mathrm{M})$ or memantine $(10 \mu \mathrm{M})$ after a 12-hour incubation in culture medium $(n=15)$. (B) The toxic effect of low levels of SSC $(1$ and $10 \mu \mathrm{M})$ was assessed using propidium iodide staining in cortical neurons after a 5 -day incubation in the absence and presence of MK801 $(1 \mu \mathrm{M})$ or memantine $(10 \mu \mathrm{M})(n=15)$. (C) Recordings in the current-clamp mode revealed a dosedependent effect of SSC on depolarization of the neuronal membrane potential. Graph represents the quantification of SSC-induced depolarization of the membrane potential. Numbers in parentheses represent the number of recorded neurons. ( $\mathbf{D}$ and $\mathbf{E})$ Efficacy of memantine treatment in the tungsten treatment study was evaluated by body weight (D) and motoric performance using rotarod testing (E) in the different mouse groups ( $n=10$ mice/group). (F) Immunoblot shows that memantine treatment decreased gephyrin degradation bands in control and tungsten-treated animals, while no degradation was observed with PSD95 ( $n=6$ mice/group). (C) Proposed sequence of events causing neurodegeneration in MoCD and SOD. Data are presented as the mean \pm SEM. ${ }^{* *} P<0.01$ and ${ }^{* * *} P<0.001$, by 2 -way ANOVA with Dunnett's multiple comparisons test (A, B, D, and E), 1-way ANOVA with Tukey's multiple comparisons test (C), or 2-tailed, unpaired Student's $t$ test (F). 
96-well tissue culture plate at a density of 30,000 cells/well, and toxicity experiments were started by the addition of different metabolites and blockers to each well, followed by an incubation step of 12 hours (unless otherwise stated) at $37^{\circ} \mathrm{C}$ in a humidified, $5 \% \mathrm{CO}_{2}$ atmosphere. Finally, cell viability was evaluated according to the supplier's protocol by measuring absorption at $570 \mathrm{~nm}$ (reference $650 \mathrm{~nm}$ ) for the MTT assay and fluorescence at $620 \mathrm{~nm}$ (excitation $530 \mathrm{~nm}$ ) for propidium iodide staining using a well plate reader (Tecan). The same procedure was used for measuring tungsten toxicity in different cells.

Live/dead staining: For discrimination between neuronal and non-neuronal toxicities, HEK293 and cortical neurons were cultivated separately on coverslips in a 24 -well plate, and cell viability in the presence of the investigated metabolites (same protocol as the MTT assay) was evaluated using a live/dead staining kit, which allows the simultaneous fluorescence-based determination of live and dead cells (Life Technologies, Thermo Fisher Scientific). Quantification was carried out in a well plate reader by measuring the fluorescence in 24-well plates at $530 \mathrm{~nm}$ (live cells, green signal measuring calcein fluorescence) and $645 \mathrm{~nm}$ (dead cells, red signal measuring ethidium homodimer-1 fluorescence) according to the supplier's protocol, and representative images were taken from the same plates using confocal laser-scanning microscopy.

Amino acid quantification and uptake. Quantification of glutamate and SSC was performed using precolumn derivatization and reversed-phase chromatography as previously described (17). For SSC quantification in protein extracts from brain, liver, or cultured neurons, metabolites were normalized to the protein amount, and for SSC quantification in urine, the determined concentrations were normalized to creatinine, which was determined using the Jaffe method as previously described (17).

Transport of the amino acids glutamate and SSC were examined in cortical neurons (DIV 10) cultured in 6-well plates at a density of 900,000 cells/well according to the following protocol: Neuronal media were removed and replaced by ACSF solution, and cells were allowed to incubate for 1 hour at $37^{\circ} \mathrm{C}$. The investigated metabolites were then added in a final concentration (if not otherwise stated) of $100 \mu \mathrm{M}$ and incubated with the cells for 1 hour at $37^{\circ} \mathrm{C}$. The ACSF medium was then removed, and cells were washed twice with ice-cold balanced salt solution (BSS) $(137 \mathrm{mM} \mathrm{NaCl}, 5.4 \mathrm{mM} \mathrm{KCl}, 10 \mathrm{mM}$ Tris/HCl, $\mathrm{pH}$ 7.6) and resuspended in $300 \mu \mathrm{l} 0.1 \mathrm{M} \mathrm{NaOH}$. After a centrifugation step for removal of cell debris $(15,000 \mathrm{~g}, 10 \mathrm{~min})$, the supernatant was divided in aliquots for determination of protein concentration and amino acid quantification using HPLC. The amount of transported metabolites was normalized to the total extracted proteins and quantified in $\mathrm{nmol} / \mathrm{mg}$ protein.

Western blot analysis. Western blotting was performed on protein crude extracts prepared either from cultivated and treated neurons or from mouse crude extracts of liver and brain derived from control and tungsten-treated animals. Protein lysates were separated by SDS-PAGE and immunoblotted using standard protocols. Membranes were probed with HRP-conjugated secondary antibodies, and signal was detected using an ECL system equipped with a cooled Chemiluminescence DeVision HQ2 camera system and Gel-Pro Analyzer software (Decon Science Tec). For band intensity quantification, the images were converted to 8-bit, the background was subtracted, and the bands were selected manually with the freehand function and automatically analyzed using ImageJ, version 1.48 $(\mathrm{NIH})$. The primary antibodies used were: anti-gephyrin (Synaptic Systems; clone 3B11; 1:50 cell culture supernatant); anti-spectrin
(Santa Cruz Biotechnology; sc-46696; 1:150); anti- $\beta$-tubulin (MilliporeSigma; T7816; 1:10,000); and anti- $\beta$-actin (MilliporeSigma; AC-47; 1:10,000). Secondary antibodies were from Santa Cruz Biotechnology and were used at 1:5,000 dilution.

Immunostaining and image processing. Immunostaining was performed for the visualization and quantification of gephyrin clusters. Neurons (DIV 12-13) were fixed with $4 \%$ paraformaldehyde and blocked with blocking solution (2\% BSA, 10\% goat serum, $0.2 \%$ Triton in PBS) for 1 hour. Primary antibodies (anti-gephyrin, clone 3B11) were applied for 1 hour in blocking solution. After 3 washing steps in PBS, neurons were incubated with secondary antibodies in 2\% BSA and $0.2 \%$ Triton for 1 hour and washed extensively with PBS, and the slides were mounted on coverslips with Fluoro gel II containing DAPI (Science Service).

Images were taken with a Nikon AZ-C2+ confocal laser-scanning microscope as a $Z$-stack of 3 optical sections with a 0.5 -mm step size. Maximum intensity projections were created and analyzed using Nikon NIS Elements 3.2 software. Regions of interest (ROIs) $(20 \times 5$ $\mathrm{mm}$ ) were placed on dendrites, and clusters were quantified using the analyze particles option in NIS Elements. Only clusters between 0.09 $\mu \mathrm{m}^{2}$ and $2 \mu \mathrm{m}^{2}$ in size were considered. For statistical analysis, values of individual ROIs were averaged, and mean values were compared for significance using a 2-tailed Student's $t$ test. Cumulative distribution of gephyrin cluster size and statistics were calculated using GraphPad Prism 6 (GraphPad Software).

Electrophysiologic recordings. Whole-cell patch-clamp recordings were performed on cultured hippocampal neurons at 15 to 23 DIV. An EPC-7 amplifier and PATCHMASTER software (HEKA) were used for the recordings. Patch pipettes, made from borosilicate glass (Science Products), had resistances of 3 to $6 \mathrm{M} \Omega$ when filled with the intracellular solution containing $130 \mathrm{mM} \mathrm{CsCl}, 5 \mathrm{mM} \mathrm{NaCl}, 0.5 \mathrm{mM} \mathrm{CaCl}$, $1 \mathrm{mM} \mathrm{MgCl}, 5$ mM EGTA, and $30 \mathrm{mM}$ HEPES (pH 7.2) was adjusted with $\mathrm{CsOH}$. The standard extracellular solution ( $\mathrm{pH}$ 7.4) contained $140 \mathrm{mM} \mathrm{NaCl}, 5 \mathrm{mM} \mathrm{KCl}, 1 \mathrm{mM} \mathrm{MgCl}, 2 \mathrm{mM} \mathrm{CaCl}_{2}, 10 \mathrm{mM}$ HEPES$\mathrm{NaOH}$, and $10 \mathrm{mM}$ glucose. Cells were recorded in voltage-clamp and current-clamp modes. For recording in voltage-clamp mode, cells were clamped at a potential of $-50 \mathrm{mV}$. Series resistances (Rs) were monitored by applying $-5 \mathrm{mV}$ voltage pulses $(50 \mathrm{~ms}$ ) every 5 seconds and ranged between $5 \mathrm{M} \Omega$ and $30 \mathrm{M} \Omega$. Experiments with a change of more than $25 \%$ in Rs throughout the recording were not included in the analyses. Cells were also recorded in current-clamp mode. In this case, the resting membrane potential of all recorded cells was determined directly after break in and ranged between -33 and $-55 \mathrm{mV}$ $(n=33)$. Series resistances $\left(\mathrm{R}_{\mathrm{s}}\right)$, monitored in voltage clamp by $-5 \mathrm{mV}$ voltage pulses ( $50 \mathrm{~ms}$ ), were between $10 \mathrm{M} \Omega$ and $40 \mathrm{M} \Omega$. For investigation of changes in membrane potential induced by SSC application, the holding current was adjusted so that the membrane potential of the neuron was at $-50 \mathrm{mV}$. Cells needing $>200 \mathrm{pA}$ current injection were not included. Depolarization of membrane potential induced by local SSC application was measured in the presence of tetrodotoxin (TTX) (0.3 $\mu \mathrm{M}$; MilliporeSigma). Data were acquired with a sampling rate of $10 \mathrm{kHz}$ after filtering at $2.8 \mathrm{kHz}$. All experiments were performed at room temperature $\left(20^{\circ} \mathrm{C}-25^{\circ} \mathrm{C}\right)$. Quantitative analysis of parameters was performed using in-house software written in IGOR 6.32A (WaveMetrics) and using Patcher's Power Tools.

Calcium imaging. Cytosolic $\mathrm{Ca}^{2+}$ dynamics were determined by ratiometric $\mathrm{Ca}^{2+}$ imaging with fura-2. The imaging set-up consisted 
of a Zeiss AxioCam/MRm CCD camera with a $1388 \times 1040$ chip and a Polychromator V (Till Photonics) that was coupled via an optical fiber to a Zeiss AxioExaminer upright microscope equipped with a $\times 40 \mathrm{~W}$ Plan Apochromat objective (numerical aperture 1). The camera and Polychromator were controlled by Axiovision software including the Physiology Module (release 4.8.2 SP3; Zeiss). During data collection, the fura-2-loaded neurons were excited with $340 \mathrm{~nm}$ or $380 \mathrm{~nm}$. Image pairs were recorded with $5.6 \mathrm{~Hz}$ and exposure times of $50 \mathrm{~ms}$ for each wavelength. Emitted fluorescence was detected through a 465 - to $555-\mathrm{nm}$ band-pass filter (BP510/90). Images were recorded in analog-to-digital units (ADUs) and stored and analyzed as 16-bit grayscale images. $\mathrm{Ca}^{2+}$ dynamics were determined from the ADU mean of the cell bodies. ROIs were defined by the soma border.

For imaging experiments, coverslips with fura-2-loaded neurons were transferred to a 1-ml recording chamber and constantly superfused with physiological saline at a flow rate of approximately $5 \mathrm{ml} / \mathrm{min}$. The saline contained $125 \mathrm{mM} \mathrm{NaCl}, 5.5 \mathrm{mM} \mathrm{KCl}, 10 \mathrm{mM} \mathrm{CaCl}_{2}, 0.8 \mathrm{mM}$ $\mathrm{MgCl}_{2}, 10 \mathrm{mM}$ HEPES, $24 \mathrm{mM}$ glucose, and $12.5 \mathrm{mM}$ sucrose, adjusted to pH 7.3 with $\mathrm{NaOH}$. Sulfinpyrazone (0.25 mM) (MilliporeSigma; S9509) was added to reduce fura- 2 sequestration. $\mathrm{Ca}^{2+}$ influx was induced by a 10-second bath application of $100 \mu \mathrm{M}$ glutamate or SSC. To test the effect of the NMDA antagonist MK801 on these responses, MK801 (10 $\mu \mathrm{M})$ was added to the imaging solution before and during the application of glutamate and SSC. Fura-2 was AM-loaded into cultured mouse cortical neurons by incubating cells with $10 \mu \mathrm{M}$ fura-2 AM (Molecular Probes; F1221) dissolved in imaging solution $\left(37^{\circ} \mathrm{C}\right.$ for $\left.30 \mathrm{~min}\right)$. Fura-2 was prepared in a stock solution ( $50 \mu \mathrm{g}$ in $5 \mu \mathrm{lDMSO})$. The final DMSO concentration in the loading solution was $0.1 \%(\mathrm{v} / \mathrm{v})$. Changes in calcium concentration were given as the normalized (to the resting state) changes in the fluorescence ratio $\left(\mathrm{R}=\mathrm{F}_{340} / \mathrm{F}_{380}\right)$. Analysis was performed offline using Axiovision and GraphPad Prism 6 (GraphPad Software).

Tungsten treatment study. Four-week-old WT C57BL/6 mice were divided into two groups of control and tungsten-treated animals. The control group ( $n=32$ ) was fed a normal diet and normal drinking water, and the tungsten-treated group $(n=32)$ received a molybdenum-free diet, and their drinking water was supplemented with $8,000 \mathrm{ppm}$ sodium tungstate, except for the initial experiments, in which different sodium tungstate concentrations were tested (100, 800, and 1,000 $\mathrm{ppm})$. Male and female mice were equally distributed between the 2 groups, and there was no discrimination between the sexes, except when comparing body weight loss between the groups. The experiment was terminated at the end of week 4 , and all mice were sacrificed and divided into small groups for different analyses.

Memantine and SSC treatment studies. For the memantine study, 4 experimental groups of 10 mice were divided into 2 control groups and 2 tungsten-treated groups, with each group receiving i.p. injections of either saline or memantine $(5 \mathrm{mg} / \mathrm{kg}$ body weight) twice a week. For the SSC study, 2 experimental groups of 10 mice were divided into 1 control and 1 SSC group receiving i.p. injections of either saline or SSC (5 mg/kg body weight) twice a week. Weekly measurement of body weight and rotarod performance was done for each mouse, and after 6 weeks, all the mice were euthanized and perfused, and the organs (liver, kidney and brain) were collected for biochemical analysis.

Rotarod testing. The rotarod instrument (Sandown Scientific; Ugo Basile, model 7650) was used to measure the motoric performance of mice. Mice were first familiarized with the task prior to testing. Mice were placed on the rotarod and allowed to acclimatize at a constant baseline speed of $3.5 \mathrm{rpm}$ before allowing the rod to accelerate to $40 \mathrm{rpm}$ for 5 minutes, with an acceleration interval of 6 seconds, and the latency to fall within this time period was recorded. Trials were conducted once per week, and data from all the trials were used to evaluate the motor coordination and balance of each mouse during the experiment.

Brain sectioning. For tissue preparation, control and tungstentreated mice were anesthetized by i.p. injection of xylazine/ketavet and perfused by intracardiac perfusion with $0.1 \mathrm{M}$ PBS, followed by $4 \%$ paraformaldehyde in $0.1 \mathrm{M}$ PBS. The mouse brains were then prepared and fixed in $4 \%$ paraformaldehyde in $0.1 \mathrm{M}$ PBS at $4^{\circ} \mathrm{C}$ overnight. Coronal sections (30- $\mu \mathrm{m}$-thick) were cut with a vibratome (Leica Biosystems), collected in ice-cold 0.1 M PBS, and stored in PBS with $0.1 \%$ sodium azide until further processing.

Nissl staining. Stored brain sections were washed with PBS, mounted on gelatine-coated slides, and air-dried for 60 minutes. The sections were then incubated for 5 minutes in $0.1 \%$ prewarmed $\left(50^{\circ} \mathrm{C}\right)$ cresyl violet solution (MilliporeSigma) and rinsed in water twice. Cresyl violet-stained sections were destained and dehydrated with ascending series of ethanol, cleared with xylene for 5 minutes, and coverslipped using Permount Mounting Medium (VWR). Slides were then scanned using the Leica SCN400 Slide Scanner.

SO activity measurements. SO activity was measured in crude liver extracts from control and tungsten-treated mice using the sulfite:cytochrome c activity assay. Briefly, $50 \mu \mathrm{g}$ crude protein extract was incubated in a $200-\mu l$ mixture (final volume) containing $50 \mathrm{mM}$ Tris/acetate, $\mathrm{pH} 8,0.2 \mathrm{mM}$ deoxycholic acid, $0.1 \mathrm{mM}$ potassium cyanide, and $0.5 \mathrm{mM}$ sodium sulfite, and the reaction was started by adding $12 \mu \mathrm{l}$ cytochrome c $(10 \mathrm{mg} / \mathrm{ml})$. SO activity was determined by monitoring the absorption change of cytochrome $\mathrm{c}$ at $550 \mathrm{~nm}\left(\varepsilon_{550}=19630 / \mathrm{M} / \mathrm{cm}\right)$. All activity was measured at room temperature $\left(25^{\circ} \mathrm{C}\right)$ using a 96 -well plate reader (BioTeK).

Statistics. All numerical data are presented as the mean \pm SEM. All experiments were performed at least 3 times, and statistical analyses were performed using GraphPad Prism 6 (GraphPad Software) with 1- or 2-way ANOVA with multiple comparisons for analysis of 3 or more groups, whereas a 2-tailed Student's $t$ test was only used for comparisons of fewer than 3 groups. For statistical analyses of the different mice groups, the median value was used, and the difference between 2 groups was considered statistically significant if the $P$ value was less than 0.05.

Study approval. All experiments involving animals complied with local and international regulations and ethics guidelines and were approved by the local research ethics committees (Germany, Landesumweltamt Nordrhein-Westfalen, reference 87-51.04.2011.A014, and Melbourne, Australia, Florey Animal Ethics Committee, reference 15-041-FINMH).

\section{Author contributions}

AK, BD, FH, MS, DF, S. Arjune, JASA, AW and S. Ayton performed the experiments and analyzed results. $\mathrm{PK}, \mathrm{JCM}, \mathrm{BD}, \mathrm{MS}, \mathrm{AIB}, \mathrm{AAB}$, and GS designed experiments, analyzed data, and reviewed the manuscript. $\mathrm{AAB}$ and GS designed the study, analyzed data, and wrote the manuscript. GS, JCM, PK, and AIB provided funding.

\section{Acknowledgments}

We gratefully acknowledge the technical assistance of Simona Jansen, Joana Stegemann, and Monika Laurien (University of 
Cologne, Institute of Biochemistry, Cologne, Germany); Helmut Wratil (University of Cologne, Institute of Zoology, Cologne, Germany); and Steve Moon, Ambili Appukuttan, and Irene Volitakis (The University of Melbourne, The Florey Institute of Neuroscience and Mental Health, Melbourne, Australia). This work was supported by the Center for Molecular Medicine Cologne (CMMC (grant D05, to GS); the Bundesministerium für Bildung und Forschung BMBF (ERA-Net Neuron II, project CIPRESS, to JCM); the Deutsche Forschungsgemeinschaft (DFG) (Priority Programme SPP 1784 ME2075/7-1, to JCM); and the National Health and Medical Research Council of Australia (AF79, to AIB). The Florey
Institute of Neuroscience and Mental Health acknowledges the substantial support received from the Victorian Government and in particular the Operational Infrastructure Support Grant.

Address correspondence to: Guenter Schwarz, Institute of Biochemistry, University of Cologne, 50674 Cologne, Germany. Phone: 49.221.470.6440; Email: gschwarz@uni-koeln.de. Or to: Abdel Ali Belaidi, The Florey Institute of Neuroscience and Mental Health, The University of Melbourne, 30 Royal Parade, Parkville, Victoria 3052, Australia. Phone: 61.3.8344.8469; Email: abdel.belaidi@florey.edu.au.
1. Johnson JL, Waud WR, Rajagopalan KV, Duran M, Beemer FA, Wadman SK. Inborn errors of molybdenum metabolism: combined deficiencies of sulfite oxidase and xanthine dehydrogenase in a patient lacking the molybdenum cofactor. Proc Natl Acad Sci U S A. 1980;77(6):3715-3719.

2. Johnson JL, Duran M. Molybdenum cofactor deficiency and isolated sulfite oxidase deficiency. In: Scriver C, Beaudet A, Sly W, Valle D, eds. The metabolic and molecular bases of inherited disease. New York, NY: McGraw-Hill; 2001:3163-3177.

3. Duran M, et al. Combined deficiency of xanthine oxidase and sulphite oxidase: a defect of molybdenum metabolism or transport? JInherit Metab Dis. 1978;1(4):175-178.

4. Reiss J, Hahnewald R. Molybdenum cofactor deficiency: Mutations in GPHN, MOCS1, and MOCS2. Human mutation. 2011;32(1):10-18.

5. Bayram E, et al. Molybdenum cofactor deficiency: Review of 12 cases (MoCD and review). European journal of paediatric neurology: EJPN: official journal of the European Paediatric Neurology Society. 2013;17(1):1-6.

6. Vijayakumar K, et al. Clinical neuroimaging features and outcome in molybdenum cofactor deficiency. Pediatric neurology. 2011;45(4):246-252.

7. Kikuchi K, Hamano S, Mochizuki H, Ichida K, Ida H. Molybdenum cofactor deficiency mimics cerebral palsy: differentiating factors for diagnosis. Pediatric neurology. 2012;47(2):147-149.

8. Waring WS, Maxwell S. Diagnosis of molybdenum cofactor deficiency. Lancet. 1999;353(9153):675-676.

9. Topcu M, Coskun T, Haliloglu G, Saatci I. Molybdenum cofactor deficiency: Report of three cases presenting as hypoxic-ischemic encephalopathy. J Child Neurol. 2001;16(4):264-270.

10. Schwarz G, Belaidi AA. Molybdenum in human health and disease. Met Ions Life Sci. 2013;13:415-450.

11. Schwarz G, Mendel RR, Ribbe MW. Molybdenum cofactors, enzymes and pathways. Nature. 2009;460(7257):839-847.

12. Klein JM, Busch JD, Potting C, Baker MJ, Langer T, Schwarz G. The mitochondrial amidoximereducing component (mARC1) is a novel signalanchored protein of the outer mitochondrial membrane. J Biol Chem. 2012;287(51):42795-42803.

13. Ryaskin OT, ed. Trends in autism research. New York, NY: Nova Biomedical Books; 2004.

14. Hartzell S, Seneff S. Impaired sulfate metabolism and epigenetics: is there a link in autism? Entro- py. 2012;14(10):1953-1977.

15. Tan WH, et al. Isolated sulfite oxidase deficiency: a case report with a novel mutation and review of the literature. Pediatrics. 2005;116(3):757-766.

16. Schwarz G. Molybdenum cofactor biosynthesis and deficiency. Cell Mol Life Sci. 2005;62(23):2792-2810.

17. Belaidi AA, Arjune S, Santamaria-Araujo JA, Sass JO, Schwarz G. Molybdenum cofactor deficiency: a new HPLC method for fast quantification of s-sulfocysteine in urine and serum. JIMD Rep. 2012;5:35-43.

18. Mechler K, Mountford WK, Hoffmann GF, Ries M. Ultra-orphan diseases: a quantitative analysis of the natural history of molybdenum cofactor deficiency. Genet Med. 2015;17(12):965-970.

19. Schwahn BC, et al. Efficacy and safety of cyclic pyranopterin monophosphate substitution in severe molybdenum cofactor deficiency type A: a prospective cohort study. Lancet. 2015;386(10007):1955-1963.

20. Lee HJ, et al. Molybdenum cofactor-deficient mice resemble the phenotype of human patients. Hum Mol Genet. 2002;11(26):3309-3317.

21. Veldman A, et al. Successful treatment of molybdenum cofactor deficiency type A with cPMP. Pediatrics. 2010;125(5):e1249-e1254.

22. Belaidi AA, Röper J, Arjune S, Krizowski S, Trifunovic A, Schwarz G. Oxygen reactivity of mammalian sulfite oxidase provides a concept for the treatment of sulfite oxidase deficiency. Biochem J. 2015;469(2):211-221.

23. Olney JW, Misra CH, de Gubareff T. Cysteine-Ssulfate: brain damaging metabolite in sulfite oxidase deficiency. J Neuropathol Exp Neurol. 1975;34(2):167-177.

24. Ye HB, Shi HB, Yin SK. Mechanisms underlying taurine protection against glutamate-induced neurotoxicity. Can J Neurol Sci. 2013;40(5):628-634.

25. Belaidi AA, Schwarz G. Molybdenum cofactor deficiency: metabolic link between taurine and S-sulfocysteine. Adv Exp Med Biol. 2013;776:13-19.

26. Zhang X, Vincent AS, Halliwell B, Wong KP. A mechanism of sulfite neurotoxicity: direct inhibition of glutamate dehydrogenase. J Biol Chem. 2004;279(41):43035-43045.

27. Huettner JE, Bean BP. Block of N-methyl-Daspartate-activated current by the anticonvulsant MK-801: selective binding to open channels. Proc Natl Acad Sci U S A. 1988;85(4):1307-1311.

28. Choi DW. Calcium and excitotoxic neuronal injury. Ann N Y Acad Sci. 1994;747:162-171.
29. Stout AK, Raphael HM, Kanterewicz BI, Klann E, Reynolds IJ. Glutamate-induced neuron death requires mitochondrial calcium uptake. $\mathrm{Nat} \mathrm{Neu}$ rosci. 1998;1(5):366-373.

30. Vergun O, Keelan J, Khodorov BI, Duchen MR Glutamate-induced mitochondrial depolarisation and perturbation of calcium homeostasis in cultured rat hippocampal neurones. JPhysiol (Lond). 1999;519 Pt 2:451-466.

31. Liu J, Liu MC, Wang KK. Physiological and pathological actions of calpains in glutamatergic neurons. Sci Signal. 2008;1(23):tr3.

32. Tyagarajan SK, Fritschy JM. Gephyrin: a master regulator of neuronal function? Nat Rev Neurosci. 2014;15(3):141-156.

33. Kobeissy FH, et al. Degradation of $\beta$ II-spectrin protein by calpain- 2 and caspase- 3 under neurotoxic and traumatic brain injury conditions. $\mathrm{Mol}$ Neurobiol. 2015;52(1):696-709.

34. Ng HK, Mahaliyana RD, Poon WS. The pathological spectrum of diffuse axonal injury in blunt head trauma: assessment with axon and myelin strains. Clin Neurol Neurosurg. 1994;96(1):24-31.

35. Park E, Liu E, Shek M, Park A, Baker AJ. Heavy neurofilament accumulation and alphaspectrin degradation accompany cerebellar white matter functional deficits following forebrain fluid percussion injury. Exp Neurol. 2007;204(1):49-57.

36. Johnson JL, Cohen HJ, Rajagopalan KV. Molecular basis of the biological function of molybdenum. Molybdenum-free sulfite oxidase from livers of tungsten-treated rats. J Biol Chem. 1974;249(16):5046-5055.

37. Johnson JL, Rajagopalan KV, Cohen HJ. Molecular basis of the biological function of molybdenum. Effect of tungsten on xanthine oxidase and sulfite oxidase in the rat. JBiol Chem. 1974;249(3):859-866.

38. Jones CM, Smith M, Henderson MJ. Reference data for cerebrospinal fluid and the utility of amino acid measurement for the diagnosis of inborn errors of metabolism. Ann Clin Biochem. 2006;43(Pt 1):63-66.

39. Dejanovic B, et al. Palmitoylation of gephyrin controls receptor clustering and plasticity of GABAergic synapses. PLoS Biol. 2014;12(7):e1001908.

40. Förstera B, et al. Irregular RNA splicing curtails postsynaptic gephyrin in the cornu ammonis of patients with epilepsy. Brain. 2010;133(Pt 12):3778-3794. 
41. Dejanovic B, et al. Exonic microdeletions of the gephyrin gene impair GABAergic synaptic inhibition in patients with idiopathic generalized epilepsy. Neurobiol Dis. 2014;67:88-96.

42. Lionel AC, et al. Rare exonic deletions implicate the synaptic organizer Gephyrin (GPHN) in risk for autism, schizophrenia and seizures. Hum Mol Genet. 2013;22(10):2055-2066.

43. Harvey RJ, Topf M, Harvey K, Rees MI. The genetics of hyperekplexia: more than startle! Trends Genet. 2008;24(9):439-447.
44. Belaidi AA, Schwarz G. Metal insertion into the molybdenum cofactor: product-substrate channelling demonstrates the functional origin of domain fusion in gephyrin. Biochem J. 2013;450(1):149-157.

45. Feng $G$, et al. Dual requirement for gephyrin in glycine receptor clustering and molybdoenzyme activity. Science. 1998;282(5392):1321-1324.

46. Winkelmann A, et al. Changes in neural network homeostasis trigger neuropsychiatric symptoms. JClin Invest. 2014;124(2):696-711.
47. Dejanovic B, et al. Simultaneous impairment of neuronal and metabolic function of mutated gephyrin in a patient with epileptic encephalopathy. EMBO Mol Med. 2015;7(12):1580-1594.

48. Thomas SJ, Grossberg GT. Memantine: a review of studies into its safety and efficacy in treating Alzheimer's disease and other dementias. Clin Interv Aging. 2009;4:367-377.

49. Kurlemann G, Debus O, Schuierer G. Dextromethorphan in molybdenum cofactor deficiency. Eur J Pediatr. 1996;155(5):422-423. 